\title{
Akteure und Strukturen in der Versorgung der Multiplen Sklerose
}

Susann Behrendt, Tonio Schönfelder, Simon Krupka, Christoph Rupprecht

\subsection{Bundesministerium für Gesundheit - 116}

6.2 Gemeinsamer Bundesausschuss - 118

6.3 Fachgesellschaften und Berufsverbände - 119

6.3.1 Medizinische und psychologische Fachgesellschaften - 119

6.3.2 Berufsverbände mit neurologischem Schwerpunkt - 121

6.4 Patientenvertretung und Selbsthilfe -122

6.4.1 Deutsche Multiple Sklerose Gesellschaft -122

6.4.2 Multiple Sklerose Selbsthilfe e.V. $\quad-123$

6.5 Individualakteure und Versorgungsmodelle - 123

6.5.1 Gesetzliche Krankenkassen - 123

6.5.2 Ärztliche und neuropsychologische Regelversorgung - 124

6.5.3 Rehabilitation und Pflege -127

6.5.4 Physiotherapie -128

6.5.5 Palliativversorgung -129

6.5.6 Pharmazeutische Unternehmen - 129

6.5.7 Patientinnen und Patienten -130

6.6 Modellprojekt: Integrierte Versorgung Multiple Sklerose am Beispiel der Region Nordrhein - 131

6.7 Forschung -134

Literatur -136 


\section{Zusammenfassung}

Eine Vielzahl von Akteuren auf unterschiedlichen Ebenen des Gesundheitssystems gestaltet die Versorgung von Patienten mit Multipler Sklerose. Das Bundesministerium für Gesundheit ist als staatlicher Akteur an der politischen und gesetzlichen Rahmensetzung beteiligt und nimmt regulatorischen Einfluss auf die Gesundheitsversorgung. Der Gemeinsame Bundesausschuss konkretisiert Leistungen für die medizinische Versorgung für die gesetzlich Versicherten. Die Leistungserbringer sowie die Ausgabenträger sind für die Implementierung und Versorgungsgestaltung verantwortlich. Aufgrund des breiten Spektrums an individuell unterschiedlichen Symptomen, Begleit- und Folgeerkrankungen sowie Behinderungen sind bereichsübergreifende Versorgungskonzepte und die Patientenorientierung bei der Versorgung von besonderer Bedeutsamkeit.

An der Therapie der Multiplen Sklerose sind Mediziner und Therapeuten unterschiedlicher Fachrichtungen und Heilberufe beteiligt. Dazu zählen neben Neurologen als Primärversorgern unter anderem Neuropsychologen, Radiologen oder Physiotherapeuten. Die Versorgungskette umfasst die ambulante und stationäre Behandlung, Rehabilitations- und Pflegeleistungen bis hin zur Palliativversorgung. Mehr als ein Drittel der Patienten mit Multipler Sklerose nimmt Leistungen aus mindestens vier Leistungsbereichen (u. a. Fachärzte, stationärer Bereich, Pflege und Arzneimittel) gleichzeitig in Anspruch. Etwa jeder fünfte Patient wird mindestens einmal pro Jahr wegen seiner Erkrankung im Krankenhaus behandelt. Regionale Untersuchungen zeigen, dass in Gegenden mit hoher Facharztdichte die Anzahl an Krankenhausaufenthalten sinkt. Modelle der integrierten Versorgung tragen dazu bei, die intersektorale Versorgung zu verbessern und die stationäre Behandlungsrate zu senken. Die Deutsche Multiple Sklerose Gesellschaft vertritt bundesweit Patienten mit Multipler Sklerose. Mit rund 45.000 Mitgliedern zählt sie zu den wichtigsten Patientenvertretungen. Neben der Etablierung von Multiple-Sklerose-Zentren zur spezialisierten Behandlung ist die Forschungsförderung eine ihrer zentralen Aufgaben. Im Zentrum der Forschungsbemühungen stehen $u$. a. die Grundlagenforschung einschließlich Krankheitsentstehung und Pathogenese der Multiplen Sklerose, neue Therapien sowie die Verbesserung der Adhärenz.
An der Versorgung von Patienten mit Multipler Sklerose (MS) ist eine Vielzahl von Akteuren beteiligt. Je nach Krankheitsstadium und -verlauf wird von der neurologischen Behandlung und hausärztlichen Versorgung über die Ergo- und Physiotherapie bis hin zur Pflege und palliativmedizinischen Begleitung ein breites Spektrum an Leistungen in Anspruch genommen. Das vorliegende Kapitel konzentriert sich angesichts dieses vielschichtigen Versorgungsgeschehens auf zentrale bundesgesundheitspolitische Akteure, Fachgesellschaften und Patientenorganisationen sowie auf jene Beteiligte, die direkt im Patientenkontakt stehen und in der MS-Forschung eine tragende Rolle spielen. Die Darstellung der Akteure und Versorgungsstrukturen orientiert sich an den verschiedenen Ebenen des deutschen Gesundheitswesens, sodass zuerst staatliche Akteure gefolgt von Institutionen der Selbstverwaltung und abschließend Individualakteure vorgestellt werden.

\subsection{Bundesministerium für Gesundheit}

Die Versorgung von Patienten mit MS in Deutschland wird in mehrerer Hinsicht auf staatlicher Ebene reguliert und beeinflusst. Das Bundesministerium für Gesundheit (BMG) ist hierbei neben den parlamentarischen Beschlussgremien Bundestag und Bundesrat der zentrale staatliche Akteur in der bundespolitischen Gestaltung des deutschen Gesundheitssystems (Busse et al. 2013). Durch Gesetzesentwürfe, Verordnungen und andere verwaltungsmäßige Vorschriften nimmt das BMG Einfluss auf gesundheitspolitische Strukturen, Prozesse und Akteure (Bundeszentrale für politische Bildung 2012). Hierbei beschlossene Regulierungen verfolgen das Ziel der Sicherstellung einer qualitativ hochwertigen, nachhaltigen, patientenorientierten und gleichzeitig wirtschaftlichen Gesundheitsversorgung. Für spezifische Indikationen, darunter schwere und seltene Erkrankungen, existieren zudem gesonderte Maßnahmen bzw. Regulierungen. $\mathrm{Zu}$ diesen spezifischen Erkrankungen gehört auch die MS. Das BMG als Fachministerium für die Kranken- und Pflegeversicherung möchte damit dem besonderen Bedarf und der Zielgenau- 
igkeit des Ressourceneinsatzes in der Versorgung Rechnung tragen.

Zur Darstellung des regulatorischen Einflusses des BMG werden nachfolgend wichtige Gesetze aufgeführt, die sich auf die Gesundheitsversorgung von Patienten mit MS auswirken. So ist das BMG maßgeblich an den Rahmenvorschriften für die Herstellung und Zulassung von Arzneimitteln und Medizinprodukten beteiligt. Mit dem Ziel einer adäquaten Arzneimittelpreisgestaltung in der gesetzlichen Krankenversicherung (GKV) erarbeitete das BMG den Entwurf zum Gesetz zur Neuordnung des Arzneimittelmarktes (AMNOG), welches 2011 in Kraft trat. ${ }^{1}$ Mit dem AMNOG wird das Ziel verfolgt, wirklichen Arzneimittelinnovationen mehr Geltung zu verschaffen. Mit Hilfe der frühen Nutzenbewertung gilt es zu bewerten, ob ein behaupteter Zusatznutzen gegenüber einer zweckmäßigen Vergleichstherapie besteht. Seither sind die pharmazeutischen Unternehmen verpflichtet, den Zusatznutzen bei Markteinführung neuer Wirkstoffe gegenüber einer festgelegten Vergleichstherapie nachzuweisen. Für vier Wirkstoffe zur MS-Therapie wurden seit 2011 Nutzenbewertungsverfahren durchgeführt ( $\triangleright$ Kap. 5).

Im GKV-Versorgungsstrukturgesetz (GKVVStG 2012) beschloss der Gesetzgeber die Neuregelung des Paragraphen $\$ 116 \mathrm{~b}$ SGB V zur ambulanten spezialfachärztlichen Versorgung (ASV). Mit dieser Gesetzesänderung können fortan nicht nur Krankenhäuser, sondern auch Vertragsärzte zur ASV von »Seltenen Erkrankungen und Erkrankungszuständen mit entsprechend geringen Fallzahlen« sowie von »schweren Verlaufsformen von Erkrankungen mit besonderen Krankheitsverläufen « sowie "hochspezialisierte Leistungen" (Gemeinsamer Bundesausschuss 2014b) zugelassen werden. $\mathrm{Zu}$ diesen Indikationen gehörte bis zur Neuregulierung 2012 auch die MS. Die Liste der Zielindikationen befindet sich gegenwärtig in Überarbeitung.

Durch das Mitte 2015 in Kraft getretene GKVVersorgungsstärkungsgesetz (GKV-VSG) soll u. a. der Zugang zu ärztlichen Leistungen gesichert werden. Die Einführung von »Terminservicestellen«

1 Gesetz zur Neuordnung des Arzneimittelmarktes in der gesetzlichen Krankenversicherung (Arzneimittelmarktneuordnungsgesetz - AMNOG) vom 22. Dezember 2010 durch die Kassenärztlichen Vereinigungen hat das Ziel, eine zeitnahe Vermittlung von Facharztterminen zu ermöglichen. Bei einer Überschreitung der Wartezeit auf einen Termin von mehr als vier Wochen kann eine Vermittlung in eine Krankenhausambulanz erfolgen. Mit dem GKV-VSG wurde darüber hinaus das Entlassungsmanagement nach einem stationären Aufenthalt neu geregelt, um einen lückenlosen Übergang in die ambulante Versorgung zu gewährleisten. Mit Entlassung aus dem Krankenhaus ist es nun möglich, dass Patienten bis zu sieben Tage Arzneimittel, Heil- und Hilfsmittel sowie Arbeitsunfähigkeitsbescheinigungen erhalten ${ }^{2}$ (DMSG 2016a).

Mit den beiden Pflegestärkungsgesetzen (PSG), die 2015 bzw. 2016 in Kraft getreten sind, wurden die Leistungen der Pflegeversicherung wesentlich erhöht. Insbesondere Unterstützungsangebote für die Pflege zu Hause wurden ausgeweitet und pflegende Angehörige werden entlastet. So können Unterstützungsleistungen wie die Verhinderungs- und Kurzzeitpflege nun besser miteinander kombiniert werden, sodass bis zu acht Wochen Kurzzeitpflege und bis zu sechs Wochen Verhinderungspflege pro Jahr möglich sind. Zudem wurden die Zuschüsse für wohnumfeldverbessernde Maßnahmen wie Rollstuhlrampen oder die Verbreiterung von Türen deutlich erhöht. Ab 2017 wird ein neuer Pflegebedürftigkeitsbegriff die bisherige Unterscheidung von Pflegebedürftigen mit körperlichen Einschränkungen und solchen mit geistigen bzw. seelischen aufheben. Ziel ist es auch, eine aktivierende Pflege mehr in den Fokus zu rücken. Die Pflegebedürftigkeit wird nun in fünf Pflegegrade eingeteilt, um Einschränkungen im Alltag differenzierter abbilden zu können. Der Grad der Selbstständigkeit einer Person wird anhand sechs pflegerelevanter Bereiche erfasst: körperliche Beweglichkeit, kognitive und kommunikative Fähigkeiten (z. B. Orientierung über Ort und Zeit), psychische Problemlagen (z. B. Ängste und Aggressionen), Möglichkeiten der Selbstversorgung (z. B. sich selbstständig ankleiden können), selbstständiger Umgang mit krankheitsund therapiebedingten Belastungen (z. B. Medika-

2 Gesetz zur Stärkung der Versorgung in der gesetzlichen Krankenversicherung (GKV-Versorgungsstärkungsgesetz GKV-VSG) vom 16. Juli 2015 
mente selbst einnehmen können) und Möglichkeiten der Gestaltung des Alltagslebens und sozialer Kontakte. Durch den neuen Pflegebedürftigkeitsbegriff wird ein erweiterter Personenkreis von rund 500.000 Menschen Zugang zu Leistungen aus der Pflegeversicherung erhalten ${ }^{3,4}$ (DMSG 2016a).

Mit dem Gesetz zur Verbesserung der Hospizund Palliativversorgung (HPG) wird die finanzielle Situation stationärer Hospize und ambulanter Hospizdienste verbessert. Krankenhäuser haben zudem die Möglichkeit, externe Palliativdienste mit der Versorgung zu beauftragen, wenn keine eigene palliativmedizinische Versorgung gewährleistet werden kann. Versicherte erhalten zudem einen Anspruch auf Beratung und Hilfestellung durch die GKV bei der Auswahl von Leistungen zur Palliativund Hospizversorgung ${ }^{5}$ (DMSG 2016a).

Patienten mit MS nehmen im Rahmen der Stufen- und symptomatischen Therapie ( $\triangleright$ Kap. 4.1 und - Kap. 4.2) häufig mehrere Arzneimittel zur gleichen Zeit ein. Im Gesetz für sichere digitale Kommunikation und Anwendungen im Gesundheitswesen (E-Health-Gesetz) wurde geregelt, dass für Menschen, die drei oder mehr Arzneimittel anwenden, ab Oktober 2016 ein Anspruch auf einen Medikationsplan besteht. Ab 2018 soll dieser Medikationsplan auch elektronisch über die Gesundheitskarte des Patienten abgerufen werden können ${ }^{6}$ (DMSG 2016a). Somit können potenzielle Arzneimittelwechselwirkungen überprüft werden.

Wichtige, das BMG beratende Gremien sind themenbezogene Komitees sowie der Sachverständigenrat zur Begutachtung der Entwicklung im Gesundheitswesen (SVR), der alle zwei Jahre ein Gutachten zu Status, Defiziten und Potenzialen im Gesundheitssystem veröffentlicht. Im aktuellen Gutachten »Bedarfsgerechte Versorgung - Perspektiven

3 Erstes Gesetz zur Stärkung der pflegerischen Versorgung und zur Änderung weiterer Vorschriften (Erstes Pflegestärkungsgesetz - PSG I) vom 17. Dezember 2014

4 Zweites Gesetz zur Stärkung der pflegerischen Versorgung und zur Änderung weiterer Vorschriften (Zweites Pflegestärkungsgesetz - PSG II) vom 21. Dezember 2015

5 Gesetz zur Verbesserung der Hospiz- und Palliativversorgung in Deutschland (Hospiz- und Palliativgesetz - HPG) vom 1. Dezember 2015

6 Gesetz für sichere digitale Kommunikation und Anwendungen im Gesundheitswesen sowie zur Änderung weiterer Gesetz vom 21. Dezember 2015 für ländliche Regionen und ausgewählte Leistungsbereiche« (2014) verweist der SVR auf Mängel der Bedarfsplanung im Sinne einer Fehlverteilung von Fachärzten für die MS-Versorgung. Damit verbunden seien teilweise lange Wegezeiten für Patienten mit MS. Erwähnt wird zudem die wachsende Bedeutung von innovativen, spezifischen Versorgungsformen und -angeboten für pflegebedürftige Patienten mit MS (Sachverständigenrat zur Begutachtung der Entwicklung im Gesundheitswesen 2014).

Die Drogenbeauftragte des Bundes ist direkt im Geschäftsbereich des BMG angesiedelt. Eines ihrer zentralen Aufgabenfelder betrifft die medizinische Verwendung von Cannabinoiden. Während das Betäubungsmittelgesetz (BtMG) das Besitzen und Erwerben von Teilen inklusive Saatgut der Hanfpflanze untersagt, können seit 2009 Sondergenehmigungen für den medizinischen Einsatz von der Bundesopiumstelle gemäß BtMG erteilt werden. Die Bundesopiumstelle ist am Bundesinstitut für Arzneimittel und Medizinprodukte, einer dem BMG nachgeordneten Bundesoberbehörde, angesiedelt. Dessen primärer Auftrag besteht in der Zulassung und Erfassung von Fertigarzneimitteln, der Gewährleistung der Arzneimittelsicherheit und der Kontrolle von Risiken bei Medizinprodukten (Bundeszentrale für politische Bildung). Das erste cannabishaltige Arzneimittel auf dem deutschen Markt zielte auf die Symptomverbesserung bei MS mit mittelschwerer bis schwerer Spastik, insofern alternative antispastische Therapien nicht (ausreichend) anschlugen (Add-on). Hierfür wurde das BtMG geändert, sodass Cannabis seit 2011 prinzipiell verschreibungsfähig ist ${ }^{7}$.

\subsection{Gemeinsamer Bundesausschuss}

Etwa 85 \% der deutschen Bevölkerung sind gegenwärtig gesetzlich krankenversichert. Demzufolge findet die gesundheitliche Versorgung überwiegend im GKV-System statt. Der Gemeinsame Bundesausschuss (G-BA) bestimmt dabei die Leistungen der medizinischen Versorgung, die im Einzelnen durch die GKV erstattet werden (Gemeinsamer Bundesausschuss 2015c).

7 25. Verordnung zur Änderung betäubungsmittelrechtlicher Vorschriften (BGBI. 2011 I S. 821) 
Der G-BA ist das höchste Beschlussgremium der gemeinsamen Selbstverwaltung, bestehend aus Krankenkassen, Ärzten und Zahnärzten, Psychotherapeuten sowie Krankenhäusern (Gemeinsamer Bundesausschuss 2015a). Die gemeinsame Selbstverwaltung ist eine Besonderheit des deutschen Gesundheitssystems. Innerhalb eines gesetzlich vorgegebenen Rahmens und unter Aufsicht von staatlichen Behörden besitzen die Selbstverwaltungspartner die Kompetenz zur konkreten Regulierung und Planung der gesundheitlichen Versorgung innerhalb des Systems der GKV (Busse et al. 2013).

Der G-BA setzt sich zusammen aus drei unparteiischen Mitgliedern und den Spitzenorganisationen der Selbstverwaltungspartner. Die Ausgabenträger werden repräsentiert durch fünf Vertreter des GKV-Spitzenverbands und die Leistungserbringer durch insgesamt fünf Vertreter der Deutschen Krankenhausgesellschaft, der Kassenärztlichen Bundesvereinigung und der Kassenzahnärztlichen Bundesvereinigung. Damit hat der G-BA insgesamt 13 stimmberechtigte Mitglieder (Gemeinsamer Bundesausschuss 2015d). An öffentlichen Sitzungen können zusätzlich fünf Vertreter von Patientenorganisationen teilnehmen. Zu ihnen gehört seit 2004 die Deutsche Multiple Sklerose Gesellschaft Bundesverband e.V. (DMSG) ( Abschn. 6.4.1) (DMSG 2012). Patientenvertreter können beratend eingreifen und Anträge stellen, besitzen jedoch kein Stimmrecht (Gemeinsamer Bundesausschuss 2015d). Insgesamt über 100 Patientenvertreter sind Teilnehmer in den G-BA-Unterausschüssen und Arbeitsgruppen.

Über das AMNOG ( $\$ 35$ a SGB V) erhielt der G-BA den Auftrag zur Durchführung einer Nutzenbewertung für alle neu zugelassenen Arzneimittel. Hierzu legen die pharmazeutischen Unternehmen dem G-BA ein Dossier vor, in dem sie den Zusatznutzen des Arzneimittels zu einer vorher festgelegten zweckmäßigen Vergleichstherapie nachweisen müssen (Gemeinsamer Bundesausschuss 2015f) ( $\triangleright$ Abschn. 5.6.4). Der G-BA kann zur Nutzenbewertung das Institut für Qualität und Wirtschaftlichkeit im Gesundheitswesen (IQWiG) oder Dritte beauftragen. Das IQWiG wurde 2004 vom G-BA gegründet und soll als unabhängige Einrichtung klinische Effektivität sowie Qualität und Wirtschaftlichkeit indikationsspezifischer medizinischer Be- handlungsverfahren evidenzbasiert evaluieren (Gemeinsamer Bundesausschuss 2010). Die Nutzenbewertung des G-BA ist eine wesentliche Grundlage der Preisbildung für das neue Arzneimittel.

Grundlage für Verhandlungen zu Erstattungsbeiträgen im Rahmen des AMNOG ist zunächst der Beschluss des G-BA über den Zusatznutzen eines neuen Arzneimittels gegenüber einer Vergleichstherapie. Patient-reported outcomes (PRO) wie Patientenpräferenzen, Patientenzufriedenheit oder wahrgenommene Krankheitssymptome finden in dem Bewertungsverfahren nur wenig oder keine Berücksichtigung. Das AMNOG kann aber eine Grundlage für eine umfassende, gesellschaftliche Diskussion über den angemessenen Preis neuer Arzneimittel schaffen. Des Weiteren ist die Datenlage insbesondere zur Arzneimittelsicherheit und Nebenwirkungen zum Zeitpunkt der frühen Nutzenbewertung nicht langfristig untersucht, sodass sich die Frage nach der Erfordernis einer längerfristigen Beobachtung stellt.

\subsection{Fachgesellschaften und Berufsverbände}

\subsubsection{Medizinische und psycholo- gische Fachgesellschaften}

MS ist eine nicht heilbare Erkrankung mit einer vielschichtigen Symptomatik und zahlreichen Begleiterkrankungen. Patienten mit dieser Krankheit bedürfen einer interdisziplinären medizinischen Versorgung. Diagnostische und therapeutische Leistungen der Neurologie, Psychologie und Psychiatrie, Neuropsychologie sowie Neuroradiologie sind hierbei primär von Bedeutung.

In Deutschland existieren für diese und andere Fachrichtungen medizinische Fachgesellschaften, die als Interessenvertretung der jeweiligen Fachärzte fungieren. Die gemeinsamen Schwerpunkte ihrer Arbeit - aus Perspektive der jeweiligen Disziplin liegen dabei auf folgenden Bereichen:

- Erstellung und Herausgabe medizinischer

Leitlinien zur evidenzbasierten Diagnostik und Therapie von Krankheitsbildern,

- Initiierung, Förderung und Vernetzung der

Forschung im jeweiligen Fachgebiet, 
- Aus- und Weiterbildung,

- Veranstaltung von nationalen und internationalen Kongressen als Plattform des wissenschaftlichen Austauschs sowie

- Aufklärungs- und Präventionsarbeit für Politik und interessierte Öffentlichkeit.

Die Deutsche Gesellschaft für Neurologie (DGN) ist die Interessenvertretung von 7.800 neurologisch tätigen Ärzten in Deutschland (Stand: 31.12.2014). Als Dachgesellschaft subsummiert die DGN verschiedene Schwerpunktgesellschaften (DGN 2015). Mit der Erkrankung MS befasst sich die Schwerpunktgesellschaft DMSG ( $\triangleright$ Abschn. 6.4.1). Die DGN ist federführender Herausgeber der aktuellen Leitlinie S2e-Leitlinie »Diagnose und Therapie der Multiplen Sklerose«. Die DMSG sowie die Bundesverbände Deutscher Nervenärzte (BVDN) und deutscher Neurologen (BDN) waren an der Erstellung als fachspezifische Institutionen beteiligt (DGN 2014).

Eine Vielzahl von psychischen Symptomen wie das Fatigue-Syndrom und die Depression können Wohlbefinden und Lebensqualität der an MS erkrankten Menschen beeinträchtigen. Diese und andere psychische Störungen stehen im Zentrum der Deutschen Gesellschaft für Psychiatrie, Psychotherapie und Nervenheilkunde (DGPPN). Sie ist die mit über 7.900 Mitgliedern größte Vereinigung für diese Fachgebiete in Deutschland (DGPPN 2015). Die DGPPN ist Herausgeber evidenzbasierter Leitlinien zur Diagnostik und Therapie unter anderen von affektiven Störungen. MS ist in den Leitlinien zur unipolaren Depression sowie zu bipolaren Störungen im neuropsychiatrischen Sinne als neurologische Grunderkrankung, primär im Bereich der differenzialdiagnostischen Abklärung, als organische Ursache von Bedeutung (DGBS e.V. u. DGPPN e.V. 2012; DGPPN et al. 2015).

Die Gesellschaft für Neuropsychologie (GNP) e.V. wurde im Jahr 1986 als gemeinnützige wissenschaftliche Fachgesellschaft von Diplom-Psychologen gegründet. Sie ist Interessenvertretung von etwa 1.500 Mitgliedern in Deutschland (Stand: 27.10.2015). Die GNP e.V. vertritt dabei die fachlichen und berufspolitischen Interessen von (Diplom-)Psychologen, die in der Forschung, in klinischen Arbeitsfeldern und im forensischen Bereich als Neuropsychologen tätig sind. Als relativ junge Spezialisierung innerhalb der Psychologie befasst sich die klinische Neuropsychologie u. a. mit Behandlungsmethoden in der Rehabilitation von Patienten mit neurologischen Erkrankungen. Seit dem Jahr 1993 besteht für Psychologen durch die GNP die Möglichkeit zur Weiterbildung zum zertifizierten Klinischen Neuropsychologen (GNP 2015b). Jedoch haben erst seit Februar 2012 durch eine gesetzliche Änderung des G-BA gesetzlich versicherte Patienten mit erworbenen neurologischen Schäden bei entsprechender Indikation einen rechtlichen Anspruch auf ambulante neuropsychologische Therapien. Die Indikation für eine neuropsychologische Therapie verlangt ein zweistufiges diagnostisches Vorgehen. Zunächst erfolgt eine somatische Abklärung durch einen Arzt, der feststellt, ob der Patient an einer erworbenen Hirnerkrankung leidet. Im zweiten Schritt erfolgen eine neuropsychologische Diagnostik, die Indikationsstellung und die Erstellung eines Behandlungsplans. Ausschließlich Psychotherapeuten oder Ärzte mit neuropsychologischer Zusatzqualifikation sind zur Durchführung von ambulanten neuropsychologischen Therapien innerhalb der vertragsärztlichen Versorgung berechtigt. Gegenwärtig ist eine flächendeckende Versorgung aus Sicht der GNP nicht gewährleistet (GNP 2015a).

Bildgebende Verfahren wie die Magnetresonanztomografie (MRT) spielen im Rahmen der frühzeitigen MS-Diagnostik eine wichtige Rolle ( $\triangleright$ Kap. 3) und werden von Neuroradiologen durchgeführt. Die Neuroradiologie gilt gemäß Weiterbildungsordnung als eine sogenannte Schwerpunktkompetenz, für die sich Ärzte mit Fachrichtung Radiologie mit mehrjähriger Weiterbildung qualifizieren können (Bundesärztekammer 2013). Die Deutsche Gesellschaft für Neuroradiologie (DGNR) repräsentiert die Interessen der neuroradiologisch tätigen Ärzte in Deutschland. Sie zählt derzeit 900 Mitglieder (DGNR 2015).

Als Dachverband aller Akteure der neurologischen Rehabilitation wurde 2005 der Bundesverband NeuroRehabilitation e.V. (BNR) gegründet. $\mathrm{Zu}$ den Mitgliedern zählen neben medizinischen Fachgesellschaften und fachbezogenen Berufsverbänden Patientenorganisationen und Selbsthilfegruppen sowie Träger entsprechender Einrichtun- 
gen. Der BNR sieht sich als Interessenvertretung der an der Neurorehabilitation Beteiligten und als Ansprechpartner politischer Akteure in fachspezifischen Fragestellungen der Neurorehabilitation (BNR 2015).

Die genannten und weitere 165 medizinische Fachgesellschaften sind in der Arbeitsgemeinschaft der Wissenschaftlichen Medizinischen Fachgesellschaften (AWMF) e.V. organisiert. Aufgrund des breiten Spektrums der (Ko-) Morbidität bei an MS Erkrankten sind viele dieser Fachgesellschaften an der Versorgung, Erforschung und Weiterbildung im Bereich der MS beteiligt. Der Überblick über MS-relevante Fachgesellschaften verzichtet an dieser Stelle auf eine vollständige und damit sehr komplexe Abbildung der beteiligten Fachdisziplinen.

\subsubsection{Berufsverbände mit neurolo- gischem Schwerpunkt}

In Deutschland existieren zwei Berufsverbände mit Fokus auf die neurologische Versorgung und ein weiterer mit Fokus auf die neuroradiologische Behandlung. Es handelt sich dabei um den Berufsverband Deutscher Nervenärzte e.V. (BVDN), den Berufsverband Deutscher Neurologen e.V. (BDN) sowie um den Berufsverband Deutscher Neuroradiologen (BDNR). Satzungsgemäß handelt es sich bei ihnen um freiwillige Zusammenschlüsse mit gemeinnützigen Aufgaben. Des Weiteren ist an dieser Stelle der Deutsche Verband für Physiotherapie (ZVK) e.V. zu nennen.

Mitglied des BVDN sind Neurologen und Nervenärzte sowie Psychiater und Psychotherapeuten mit Wohnsitz in der Bundesrepublik. Der BVDN versteht sich als Repräsentanz der ambulant tätigen Ärzte mit den genannten Fachrichtungen (BVDN 2015a). Demgegenüber ist der BDN die berufspolitische Interessenvertretung der neurologisch tätigen niedergelassenen und stationär arbeitenden Ärzte (BDN 2015a). Er wurde auf Initiative der DGN sowie des BVDN, Sektion Neurologie, gegründet und ist ausschließlich auf die Fachrichtung Neurologie ausgerichtet. Das berufspolitische Organ der neuroradiologischen Ärzte in Deutschland ist der BDNR und wurde 1982 als gemeinnütziger
Verein eingetragen. Satzungsgemäß sind hier nur Ärzte Mitglied, die mindestens drei Jahre hauptberuflich neuroradiologisch tätig waren. Zudem weisen Mitglieder eine fachspezifische Ausbildung an einer neuroradiologischen Einrichtung vor, die von einem hauptamtlich tätigen Neuroradiologen geleitet wird (BDNR 2015).

BDN, BVDN und BDNR streben an, die Versorgung in den jeweiligen Versorgungssettings sicherzustellen, ihre Mitglieder bei fachlichen, rechtlichen oder ökonomischen Fragen zu beraten sowie die Entwicklung von sektorenübergreifenden Behandlungsoptionen voranzutreiben. Sie repräsentieren zudem ihre Mitglieder gegenüber relevanten Institutionen wie der Bundesärztekammer oder der Kassenärztlichen Bundesvereinigung. Seit 2001 existiert eine Fortbildungsakademie, die vom BDN, BVDN sowie vom Berufsverband Deutscher Psychiater e.V. (BVDP) gegründet wurde. Zielgruppe der Veranstaltungen sind niedergelassene Nervenärzte, Neurologen und Psychiater bzw. Psychotherapeuten (BDN 2015b).

Im Jahr 2002 gründeten der BVDN und der $\mathrm{BDN}$ den Bundesverband ambulante/teilstationäre Neurorehabilitation (BV ANR e.V.). Dessen ausgewiesenes Ziel ist es, Versorgungspfade von neurologischen Patienten von der akuten Behandlung über die Rehabilitation bis hin zur Nachsorge beim niedergelassenen Arzt zu optimieren (BDN 2015c). So unterstreicht der BDN öffentlich den Bedarf an integrierten Modellen der MS-Behandlung innerhalb der GKV und fordert mehr Anreize zur sektorenübergreifenden flächendeckenden Versorgung.

Der Dachverband des BDN, des BVDN, des BVDP sowie des Berufsverbands für Kinder- und Jugendlichen-Psychiatrie und -Psychotherapie (BKJPP) ist seit 2013 der Spitzenverband ZNS (SPiZ). Er befasst sich insbesondere mit den bestehenden und zukünftigen Herausforderungen der Versorgung von Menschen mit Erkrankungen des Zentralnervensystems (ZNS), artikuliert Defizite und Verbesserungspotenzial und kommentiert gesundheitspolitische Entwicklungen in Deutschland (BVDN 2015b).

Der Deutsche Verband für Physiotherapie (ZVK) e.V. ist der führende Interessenverbund der Physiotherapeuten. Seine Aufgabe besteht in der Positionierung und Entwicklung des Berufsstandes 
im Rahmen der gesellschaftlich und gesundheitsökonomischen Entwicklung (Deutscher Verband für Physiotherapie (ZVK) e.V. 2015b). Er besteht aus einem Bundesverband sowie 13 Landesverbänden. Mitglieder können sowohl angestellte als auch freiberuflich tätige Physiotherapeuten werden. Der Verband unterhält verschiedene Arbeitsgruppen, beispielsweise in den Bereichen Bobath-, PNF- und Vojta-Therapie. Die Arbeitsgruppen sind zuständig für Beratungen, Zertifizierungsfragen, Fortbildungen sowie aktuell auch für die Entwicklung von Assessmentverfahren in den entsprechenden Bereichen (Deutscher Verband für Physiotherapie (ZVK) e.V. 2015a).

\subsection{Patientenvertretung und Selbsthilfe}

\subsubsection{Deutsche Multiple Sklerose Gesellschaft}

Im Gegensatz zu anderen Schwerpunktgesellschaften der DGN handelt es sich bei der DMSG um eine Patientenorganisation. Sie existiert seit mehr als 60 Jahren. Im Jahr 2015 waren unter ihrem Dach in 16 Landesverbänden mit rund 900 Kontaktgruppen 4.200 Mitarbeiter ehrenamtlich aktiv. Mit ca. 250 hauptamtlichen Sozialarbeitern, Psychologen und Mitarbeitern aus anderen therapeutischen Feldern bietet die DMSG Unterstützung für die Selbsthilfe, aber auch individuelle Einzelberatung an. Insgesamt zählte die DMSG 2014 knapp 45.000 Mitglieder bundesweit (DMSG 2015c), die fast ausschließlich an MS Erkrankte und Angehörige sind. Sie finanziert ihre Arbeit primär aus Spenden, Erbschaften, Mitgliedsbeiträgen, Zuschüssen von Stiftungen und zu einem geringen Teil durch finanzielle Unterstützung der Deutschen Rentenversicherung, von Krankenkassen sowie wenigen zweckgebundenen Zuwendungen pharmazeutischer Unternehmen.

Zielgruppen ihres Wirkens sind zum einen an MS Erkrankte selbst und deren Angehörige, zum anderen die Leistungserbringer des Versorgungsgeschehens und die interessierte Öffentlichkeit. Sowohl auf Bundes- als auch auf Landesebene der DMSG wird die fachliche Arbeit durch den Ärztli- chen Beirat mit Experten aus der MS-Forschung und MS-Versorgung sowie den Bundesbeirat MS Erkrankter unterstützt, der sich aus an MS erkrankten Personen zusammensetzt.

Das Aufgabenspektrum der DMSG teilt sich in folgende zentrale Bereiche (DMSG 2015a):

- Forschungsförderung

- Aufklärung, Information, Fortbildung und Beratung

- versorgungskonzeptionelle Tätigkeiten mit dem Schwerpunkt »sozialmedizinische Nachsorge"

- Interessenvertretung der ca. 200.000 an MS Erkrankten in Deutschland

Seit 2001 erfasst die MS Forschungs- und Projektentwicklungs-gGmbH, eine Tochtergesellschaft der vom DMSG Bundesverband gegründeten Deutschen MS Stiftung, bundesweit epidemiologische und behandlungsrelevante Daten von an MS Erkrankten und führt diese in einem Datensatz zusammen ( $\triangleright$ Kap. 4.1.3). Der Bundesverband der DMSG engagiert sich zudem im Bereich der Ausund Weiterbildung von medizinischen Fachkräften in der MS-Therapie und -Pflege. Beispiele sind berufsbegleitende Fortbildungen zur »MS-Schwester « und in der ambulanten und stationären Pflege.

Die Etablierung von MS-Kompetenzzentren, d.h. die Entwicklung der DMSG-Zertifikate »MSZentren «, "MS-Schwerpunktzentren « und »MSRehabilitationszentren « für auf MS-spezialisierte Einrichtungen dient maßgeblich der Orientierung von an MS Erkrankten durch entsprechende Qualitätskriterien und soll den Patienten Anhaltspunkte geben, wo ihre Erkrankung spezialisiert behandelt werden kann. Die Website des Bundesverbandes der DMSG (www.dmsg.de) enthält neben vielen relevanten Informationen auch die Adressen dieser ausgezeichneten Zentren. Hierzu zählen Akutkliniken mit Zulassung gemäß $\$ 108$ SGB V, Rehabilitationskliniken mit Zulassung gemäß $\$ 111$ SGB V, Universitätskliniken sowie niedergelassene Schwerpunkt- bzw. neurologische Praxen (DMSG 2015e). Die Anerkennungskriterien, vom Ärztlichen Beirat der DMSG definiert, sind insbesondere:

- Mindestanzahl betreuter MS-Patienten,

- DMSG-standardisierte Diagnostik und Dokumentation der Befunde, 
- Therapie gemäß Leitlinien (Multiple Sklerose Therapie Konsensus Gruppe (MSTKG)

(DMSG 2016b).

Da die Teilnahme am oben beschriebenen DMSGDatensatz für das Zertifikat als MS-Zentrum verpflichtend ist, geben die gesammelten Informationen der Teilnehmer Auskunft über diese MS-Versorgungslandschaft. Aktuell (Stand: 22.01.2016) handelt es sich um 164 Einrichtungen. Davon sind $47 \%(\mathrm{n}=77)$ MS-Schwerpunktzentren, $42 \%(\mathrm{n}=69)$ MS-Zentren und $11 \%(\mathrm{n}=18)$ MS-Rehabilitationszentren (DMSG 2016b).

Seit 1997 bietet der DMSG Bundesverband neben Fortbildungen für Physiotherapeuten und MS Schwestern auch Angebote zur »Pflege bei MS« an . Zielgruppe sind Mitarbeiter ambulanter Pflegedienste bzw. stationärer Pflegeeinrichtungen. Pflegeeinrichtungen mit mindestens zwei fortgebildeten Mitarbeitern können ein DMSG-Zertifikat mit dreijähriger Laufzeit erhalten (DMSG-geprüfter Pflegedienst bzw. Pflegestation) (DMSG 2015b).

Die Landesverbände der DMSG sind für die Beratung von Erkrankten und Angehörigen, für die Bereitstellung und Organisation alltagsrelevanter Unterstützungsleistungen (wie z.B. Fahrdienste, Selbsthilfe und Rollstuhl-Mobilitätstrainings) sowie für Fortbildungsveranstaltungen (wie Patientenschulungen und Tagungen) zuständig. Zudem übernehmen Landesverbände auch Trägerschaften, beispielsweise für betreute Wohngruppen, und sind an Kliniken mit MS-Schwerpunkt beteiligt. Solche Gesellschaftsanteile an Spezialkliniken werden von den Landesverbänden der DMSG in Bayern und Baden-Württemberg (AMSEL) gehalten. Die Landesverbände der DMSG sind wichtige Plattformen bei der Organisation und Vernetzung des sogenannten Peer Counseling. An MS Erkrankte, als Peer Counselor geschulte Menschen, beraten dabei andere MS-Betroffene als eine Form der Selbsthilfe, die der internationalen Independent Life-Bewegung entlehnt ist und einen Beitrag zum selbstbestimmten, eigenverantwortlichen Leben für Menschen mit Behinderung leisten soll. Im Zentrum der Unterstützung stehen dabei psychosoziale Hilfestellungen (DMSG 2015d)

\subsubsection{Multiple Sklerose Selbsthilfe e.V.}

Die Multiple Sklerose Selbsthilfe e.V. widmet ihre Arbeit als gemeinnützige und unabhängige Institution seit ihrer Gründung 1981 den Erfahrungen und Perspektiven von MS-Betroffenen und bietet Unterstützung bei der persönlichen Krankheitsbewältigung, Informationen zu Therapien und Einrichtungen, aber ebenso praktische Hilfen im Umgang mit Versicherungen und Antragstellungen. Vierteljährlich publiziert die Multiple Sklerose Selbsthilfe e.V. die Zeitschrift »Blickpunkt», welche sich primär an Betroffene richtet und über MS-relevante medizinische Erkenntnisse, aber ebenso über aktuelle Rechtsprechungen mit Bezug zu MS, informiert (MSK 2015).

\subsection{Individualakteure und Versorgungsmodelle}

\subsubsection{Gesetzliche Krankenkassen}

Die komplexe Versorgung von Patienten mit MS unter Einbezug einer Vielzahl von Leistungserbringern und des Einsatzes verschiedener therapeutischer Strategien in unterschiedlichen Settings stellt für die gesetzlichen Krankenkassen mehr eine versorgungsstrukturelle als eine finanzielle Herausforderung dar. Patienten mit MS benötigen intensive ärztliche ambulante und stationäre Behandlungen, Krankengeld, Arzneimittel sowie Heil- und Hilfsmittelverordnungen. Hinzu kommen die Inanspruchnahme von Rehabilitations- und Pflegeleistungen und der Bedarf nach sozialen Hilfestrukturen. Hierdurch ergibt sich u.a. der Bedarf an innovativen Behandlungsprogrammen (Schlenker 2014).

\section{- Integrierte Versorgung}

Nach $₫ 140$ a SGB V sind Krankenkassen berechtigt, Verträge zur sektorenübergreifenden bzw. interdisziplinären Versorgung, d.h. zur integrierten Versorgung (IV) ihrer Versicherten abzuschließen. Vertragspartner sind primär vertragsärztliche Leistungserbringer sowie Krankenhäuser mit Versorgungsauftrag, aber auch pharmazeutische Unternehmen ${ }^{8}$.

8 § 104b Abs. 1 SGB V 
Für die Versicherten handelt es sich um ein Angebot, an dem sie freiwillig teilnehmen können ${ }^{9}$.

Integrierte Versorgungsmodelle sind Suchprozesse oder »Testfelder« für eine bessere Gesundheitsversorgung, mit dem Zweck fragmentierte Behandlungsverläufe durch ein stimmiges und qualitätsgesichertes Gesamtkonzept zu ersetzen. Solche Konzepte umfassen eine schnelle und präzise Indikationsstellung, eine adäquate Therapie genauso wie entsprechende Hilfestellungen im psychosozialen Bereich (Amelung 2010).

Für Patienten mit MS existiert bisher bundesweit ein IV-Modell in Deutschland. Als Kooperation mit dem BDN und der DMSG initiierte die AOK Rheinland/Hamburg 2006 ein Modell zur MSIntegrationsversorgung. Ziele des IGV-MS Rheinland sind die Optimierung der Zusammenarbeit von ambulanten und stationären Leistungserbringern, die Reduktion der stationären Aufenthalte von Patienten mit MS sowie die Verbesserung des Erkrankungsverlaufs (Schlingensiepen 2010). Mittlerweile haben weitere gesetzliche Krankenkassen diesen IV-Vertrag unterzeichnet (Schlingensiepen 2014). Mehr als 120 niedergelassene Neurologen und Nervenärzte sowie 14 Kliniken sind involviert und erhalten je nach erbrachter Leistung von den Krankenkassen eine zusätzliche Vergütung. Knapp 2.000 Patienten mit MS nehmen an der Versorgung teil (Landeszentrum Gesundheit Nordrhein-Westfalen 2015) ( $>$ Abschn. 6.6).

Zwischen 2005 und 2009 kooperierte zudem die BARMER GEK mit der Universität Gießen im Rahmen eines Selektivvertrags zur MS-Versorgung. Die Programmevaluation ergab eine gesteigerte $\mathrm{Zu}$ friedenheit der Patienten, jedoch keine wirtschaftlichere Versorgung und bisher keinen Nachweis der Steigerung der Lebensqualität (Schlenker 2014). Um Ausbildung und Einsatz von ambulant tätigen Versorgungsassistenten mit Schwerpunkt Neurologie und Psychiatrie (Neuro-EVA bzw. EVA-NP) zu fördern, schlossen die BARMER GEK NordrheinWestfalen und die DAK-Gesundheit 2014 einen Versorgungsvertrag mit der KV Westfalen-Lippe. Zielindikationen sind neben anderen Erkrankungen die MS (Anders u. Oschmann 2006; Schlenker 2014). Diese neue Maßnahme zur Verbesserung der neurologischen Versorgung soll insbesondere Neurologen bei ihrer Arbeit entlasten (BARMER GEK NRW et al. 2014).

\subsection{2 Ärztliche und neuropsycholo- gische Regelversorgung}

Das Ziel der medizinischen Leistungserbringung bei Patienten mit MS besteht darin, den Betroffenen eine möglichst hohe Lebensqualität, Mobilität und Selbstbestimmung zu ermöglichen und zu erhalten. Aufgrund der Symptomvielfalt der Erkrankung stehen viele unterschiedliche medizinische Akteure im direkten Patientenkontakt. Sie nehmen zusammen mit dem Patienten unmittelbar Einfluss auf Versorgungsprozesse, -qualität und -outcome. Je nach Erkrankungsverlauf können eine Akutbehandlung im Krankenhaus, stationäre bzw. rehabilitative Maßnahmen oder/und eine ambulante Therapie und langfristige Nachsorge indiziert sein (Deutsche Gesellschaft für Neurologie und Kompetenznetz Multiple Sklerose 2014). Die Versorgung von Patienten mit MS bedarf demzufolge einer effektiven Schnittstellenkommunikation innerhalb und zwischen den einzelnen Sektoren (Weegen et al. 2013; Windt 2014).

Viele Ärzte unterschiedlicher Fachrichtungen sind versorgungsrelevante Akteure der ambulanten ärztlichen Versorgung von Menschen mit MS. Die Primärversorgung erfolgt durch Neurologen. Rund $83 \%$ der Patienten werden ambulant aufgrund ihrer MS-Erkrankung ausschließlich von Ärzten der Fachgruppen Neurologie, Nervenheilkunde und Psychiatrie behandelt (Stand: 2010) (IGES Institut 2014). Weitere relevante Akteure der ambulanten ärztlichen Versorgung sind zudem Neuroradiologen und Hausärzte. Potenzielle MS-Begleit- und Folgeerkrankungen erfordern überdies den Einbezug von weiteren Facharztgruppen, u. a. von Urologen und Augenärzten. Die Herausforderung in der Therapieentscheidung besteht für die Ärzte u. a. darin, Qualität der Versorgung (Bundesärztekammer 2015) und Wirtschaftlichkeit ${ }^{10}$ miteinander in Einklang zu bringen.

Die klinische Neuropsychologie versucht Art und Schweregrad von Beeinträchtigungen kogniti-

$10 \S 12 \mathrm{SGB} V$ 


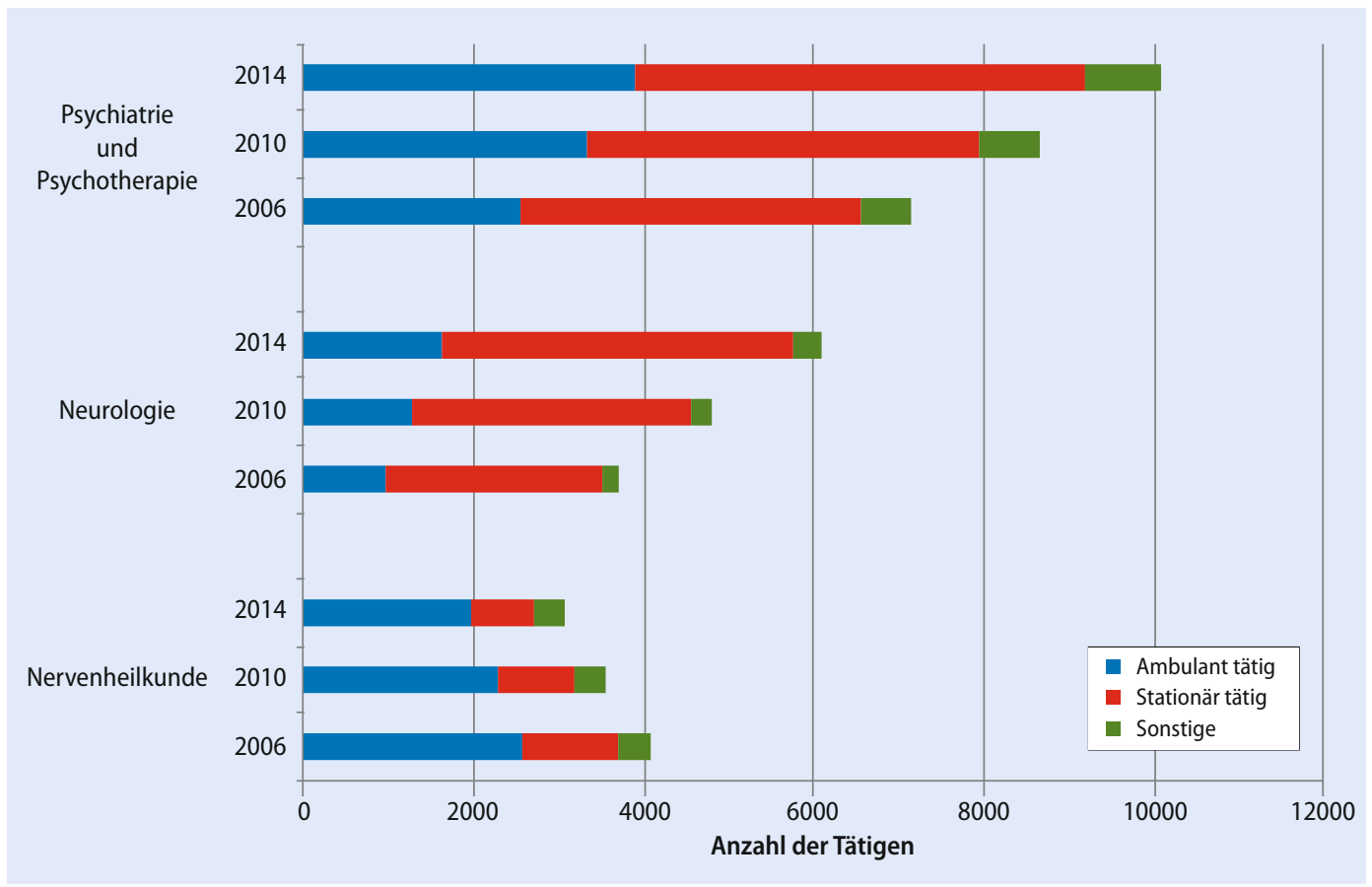

- Abb. 6.1 Fachärzte der Nervenheilkunde, Neurologie sowie der Psychiatrie und Psychotherapie in Deutschland: Anzahl der Ärzte 2006, 2010 und 2014

Quelle: IGES - Bundesärztekammer (2007, 2011, 2014)

Anmerkung: inklusive Ärzte, die in Körperschaften, Behörden und sonstigen Einrichtungen tätig waren

ver Funktionen festzustellen und durch neuropsychologische Verfahren zu behandeln. Seit dem Jahr 2012 gehört die neuropsychologische Therapie zur vertragsärztlichen Versorgung. Die Therapie ist inzwischen nicht mehr antragspflichtig. Lediglich der Beginn der Behandlung ist der Krankenkasse anzuzeigen (KBV 2012). Seit dem Jahr 2013 sind diese Leistungen Bestandteil des Einheitlichen Bewertungsmaßstabs (EBM) und müssen daher nicht mehr zur Kostenerstattung vom Patienten bei der gesetzlichen Krankenkasse eingereicht werden.

Die Zahl der Neurologen in Deutschland ist in den letzten Jahren stark gestiegen (• Abb. 6.1). Wie Daten der Bundesärztekammer veranschaulichen, gibt es zunehmend mehr Fachärzte der Neurologie $(+64,4 \%)$ sowie Psychiatrie und Psychotherapie $(+41,13 \%)$. Die Zahl berufstätiger Neurologen stieg seit 2006 von rund 3.700 bis 2014 auf rund 6.100 Ärzte an. Die Zahl der berufstätigen Fachärzte für Psychiatrie und Psychotherapie erhöhte sich in diesem Zeitraum von rund 7.000 auf rund 10.000 .
Gleichzeitig ist die Zahl der Nervenärzte (Fachärzte der Nervenheilkunde) rückläufig (-24,8 \%) (Bundesärztekammer 2007, 2011, 2015). Dieser Trend zeigt sich einheitlich für den ambulanten und stationären Bereich der Versorgung. Die Abnahme der Anzahl von Nervenärzten ist primär strukturell bedingt, weil seit Inkrafttreten der (Muster-) Weiterbildungsordnung im Jahr 2003 diese Facharztanerkennung nicht mehr erlangt werden kann (Bundesärztekammer 2013). Die neu eingeführte Bezeichnung Facharzt für Psychiatrie und Psychotherapie ersetzte den Facharzt für Psychiatrie und Nervenarzt.

Die Verteilung von MS-relevanten Facharztpraxen in der Bundesrepublik variiert regional. Dass sich ein Zusammenhang zwischen der diesbezüglichen Praxisdichte und der Behandlungsqualität bzw. -kontinuität beobachten lässt, zeigte eine erst kürzlich publizierte Studie auf Basis von Daten der BARMER GEK. Auftraggeber der Studie waren die KBV, die DGN sowie Berufsverbände der Nerven- 
ärzte, Neurologen und der Psychiater ${ }^{11}$. Nahezu jeder Patient mit MS (98,8 \% der eingeschlossenen 19.496 Patienten mit MS) hatte der Studie zufolge im Jahr 2010 mindestens einen Kontakt zu einem ambulanten Vertragsarzt (Behandlungsfälle mit und ohne MS-Diagnose). 88,4 \% der Patienten mit MS wurden durch ambulant tätige vertragsärztliche Neurologen, Nervenärzte bzw. Psychiater behandelt (Behandlungsfälle mit MS-Diagnose). Die MS-Behandlung erfolgte bei $83,3 \%$ der Patienten ausschließlich, d.h. ohne Beteiligung bzw. Überweisung des Hausarztes, bei den genannten Fachgruppen (IGES Institut 2014).

Die inter- bzw. intrasektorale Versorgung bei MS belief sich im Zeitraum 2008 bis 2010 gemäß der Studie primär auf die Kombination von Hausarzt, Facharzt und Arzneimittelversorgung (bei 40,2\% der Patienten). Rund ein Drittel (36\%) der Patienten nahm Leistungen aus mindestens vier Leistungsbereichen in Anspruch; neben den bereits genannten Sektoren handelte es sich dabei insbesondere um den stationären Bereich und Pflege. Aufgrund der Inanspruchnahme von Leistungen aus vielen verschiedenen Sektoren durch Patienten mit MS bieten sich Integrierte Versorgungsmodelle an, um Verbesserungen in der Versorgungserbringung für diese Patientengruppen zu erzielen ( $\triangleright$ Abschn. 6.6).

Die Facharztdichte ist in Ballungsgebieten im Vergleich zu ländlichen Gegenden höher. Allerdings ist diese Konzentration von Fachärzten kein alleiniges Qualitätsmerkmal der Versorgung. So ist zu beachten, dass entsprechend den Bedarfsplänen der Kassenärztlichen Vereinigungen und Landesverbände der Krankenkassen Fachärzte in großflächige Planungsbereiche eingeteilt werden und damit auch für die Versorgung größerer Räume zuständig sind (IGES Institut 2014).

Zwischen regionaler ambulanter Facharztdichte und der Anzahl stationärer Aufenthalte ließ sich ein inverser Zusammenhang feststellen (• Abb. 6.2). Diese Ergebnisse implizieren, dass Versorgungskapazitäten die Qualität der MS-Versorgung mitbe-

11 Die Auswahl der betrachteten Facharztgruppen erklärt sich über die untersuchten Indikationen: MS, Demenz und Schizophrenie. http://www.aerzteblatt.de/nachrichten/60690/Je-hoeher-die-Facharztdichte-desto-besserdie-Versorgung stimmen und betonen den Bedarf einer verbesserten Schnittstellenkommunikation im Versorgungssystem (IGES Institut 2014).

\section{- Ambulante spezialfachärztliche Versorgung}

Die ASV beinhaltet die Diagnostik und Behandlung komplexer, schwer zu therapierender Erkrankungen, die eine spezielle Qualifikation, eine interdisziplinäre Zusammenarbeit und besondere Ausstattungen erfordern. Hierzu gehören Erkrankungen mit besonderen Krankheitsverläufen, seltene Erkrankungen und Erkrankungszustände mit entsprechend geringen Fallzahlen sowie hochspezialisierte Leistungen ( $\$ 116 \mathrm{~b}$ SGB V). Ziel der ASV ist die Schaffung eines eigenständigen ambulanten Versorgungsbereiches. Insbesondere Patienten mit komplexen oder ungewöhnlichen Verläufen der MS und einer hohen Variation an Symptomen könnten von einem Ausbau der ASV, in denen Behandlungsteams mit psychologischer und neurologischer Kompetenz eine interdisziplinäre, symptomatische Behandlung durchführen, profitieren. Gegenwärtig wird die Liste der Zielindikationen vom G-BA schrittweise überarbeitet. In der ASV liegen zurzeit erst für zwei Indikationen abschließende Regelungen durch den G-BA vor. Insgesamt gibt es nur wenige zugelassene interdisziplinäre ASV-Teams, was u. a. auf den bürokratischen Aufwand zur Implementierung eines solchen Teams zurückzuführen ist. So ist z. B. noch umstritten, auf welche Weise Krankenhäuser Fachkundenachweise für ihre Ärzte vorlegen müssen (Gerst 2015).

Im Rahmen der ASV nach \$116b SGB V können sowohl niedergelassene Vertragsärzte als auch Krankenhäuser seit der Gesetzesänderung im Jahr 2012 unter bestimmten Voraussetzungen eine $\mathrm{Zu}$ lassung für die ASV erhalten ( $\triangleright$ Abschn. 6.1). Für Krankenhäuser bestand die Möglichkeit zur ASV bereits seit 2004 und bezog sich unter anderem auf die MS (\$116b Abs. 3 (alt) SGB V). Informationen zum aktuellen bundesweiten Stand der bisher erteilten ASV-Zulassungen sind bisher nicht verfügbar. Wenige Bundesländer veröffentlichen Informationen zur Anzahl von für die ASV registrierten Einrichtungen. Die Angaben beziehen sich jedoch auf den Zeitraum vor Novellierung des $\$ 116 b$ SGB V. So waren beispielsweise in Sachsen und ebenso in Schleswig-Holstein im Jahr 2011 jeweils neun Kran- 


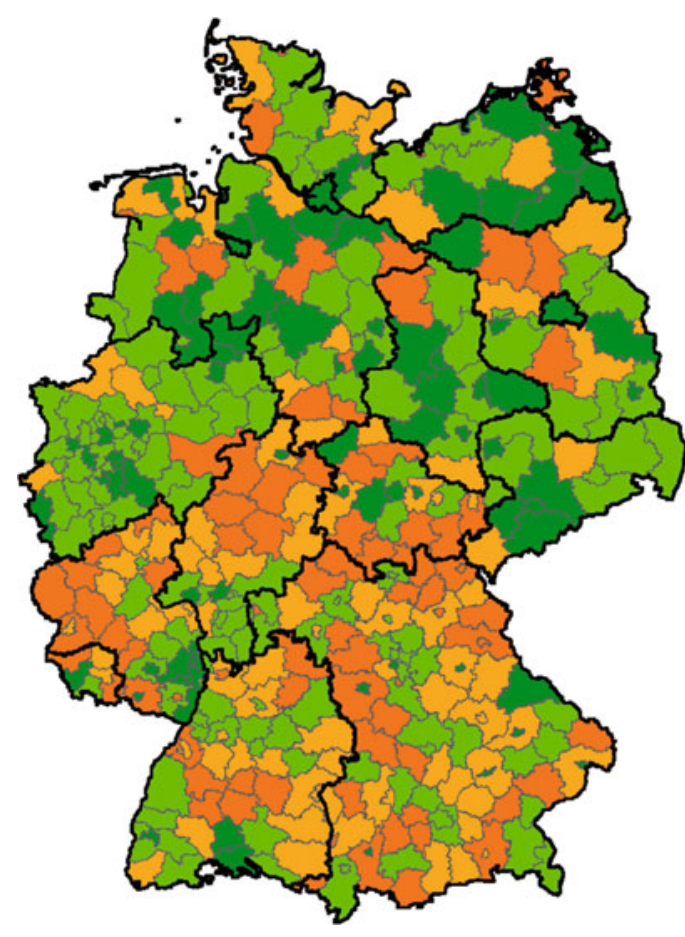

Anteil MS-Patienten mit ausschließlich fachärztlicher Versorgung nach Stadt- und Landkreisen

- $<70 \% \quad \geq 70 \%-80 \% \quad \square \geq 80 \%-90 \% \quad \square \geq 90 \%-100 \%$

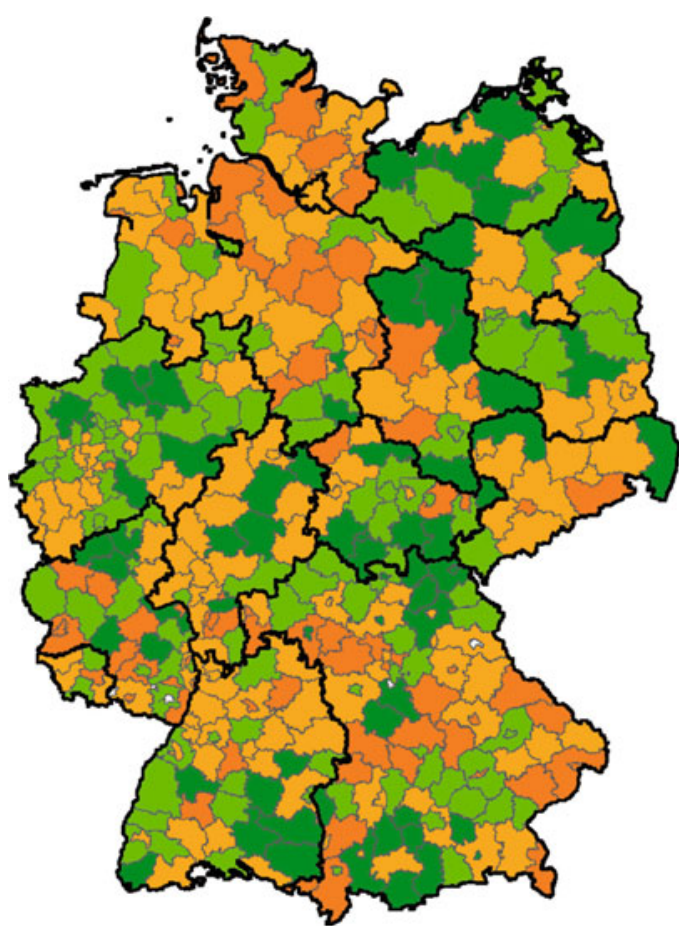

Anteil MS-Patienten mit mind. einem krankheitsspezifischen $\mathrm{KH}$-Aufenthalt nach Stadt- und Landkreisen

$\square<10 \% \quad \geq 10 \%-20 \% \square \geq 20 \%-30 \% \quad \square \geq 30 \%-65 \%$

- Abb. 6.2 Regionale Unterschiede in der MS-Versorgung: ambulante Facharztdichte und stationäre Inanspruchnahme Quelle: IGES - Routinedaten BARMER GEK (IGES Institut 2014)

kenhäuser für die ASV der MS zugelassen (Landesportal Schleswig-Holstein 2015; Staatsministerium für Soziales und Verbraucherschutz Freistaat Sachsen 2011). In Berlin nahmen bis April 2013 drei Krankenhäuser an dieser MS-Versorgungsform teil (Senatsverwaltung für Gesundheit und Soziales Berlin 2013).

\subsubsection{Rehabilitation und Pflege}

Die medizinische Rehabilitation kann u. a. ärztliche, psychologische sowie andere heilberufliche Therapien, Arzneimittel, Heil- und Hilfsmittel und die Ergotherapie umfassen. Neben der medizinischen Rehabilitation gibt es noch weitere Formen der Rehabilitation: die Leistungen zur Teilhabe am Arbeitsleben (LTA) und die soziale Rehabilitation (Augurzky et al. 2011).
Im Rahmen der (multimodalen) Rehabilitation unterstützen die jeweiligen Akteure den Patienten mit MS bei dem Erhalt seiner Mobilität, der Teilhabe am sozialen Leben und Berufsleben und insbesondere bei der Erleichterung des beruflichen Wiedereinstiegs ( $\triangleright$ Kap. 4). Das mittlere Alter bei Krankheitsbeginn beträgt rund 31 Jahre (Flachenecker et al. 2008). Ein großer Anteil der volkswirtschaftlichen Kosten durch MS entfällt auf indirekte Kosten durch Produktivitätsausfälle aufgrund von Arbeits- und Erwerbsunfähigkeit ( $\triangleright$ Abschn. 5.3). So beziehen rund $40 \%$ der Patienten mit MS eine Erwerbsminderungsrente, nur circa $28 \%$ sind voll berufsfähig und $9 \%$ teilzeitbeschäftigt (Flachenecker et al. 2008).

Die medizinische Rehabilitation ist nach SGB IX $\$ 19$ geregelt und wird stationär, ambulant oder mobil durchgeführt. Die stationäre Rehabilitation erfolgt überwiegend bei Patienten mit höheren motorischen 


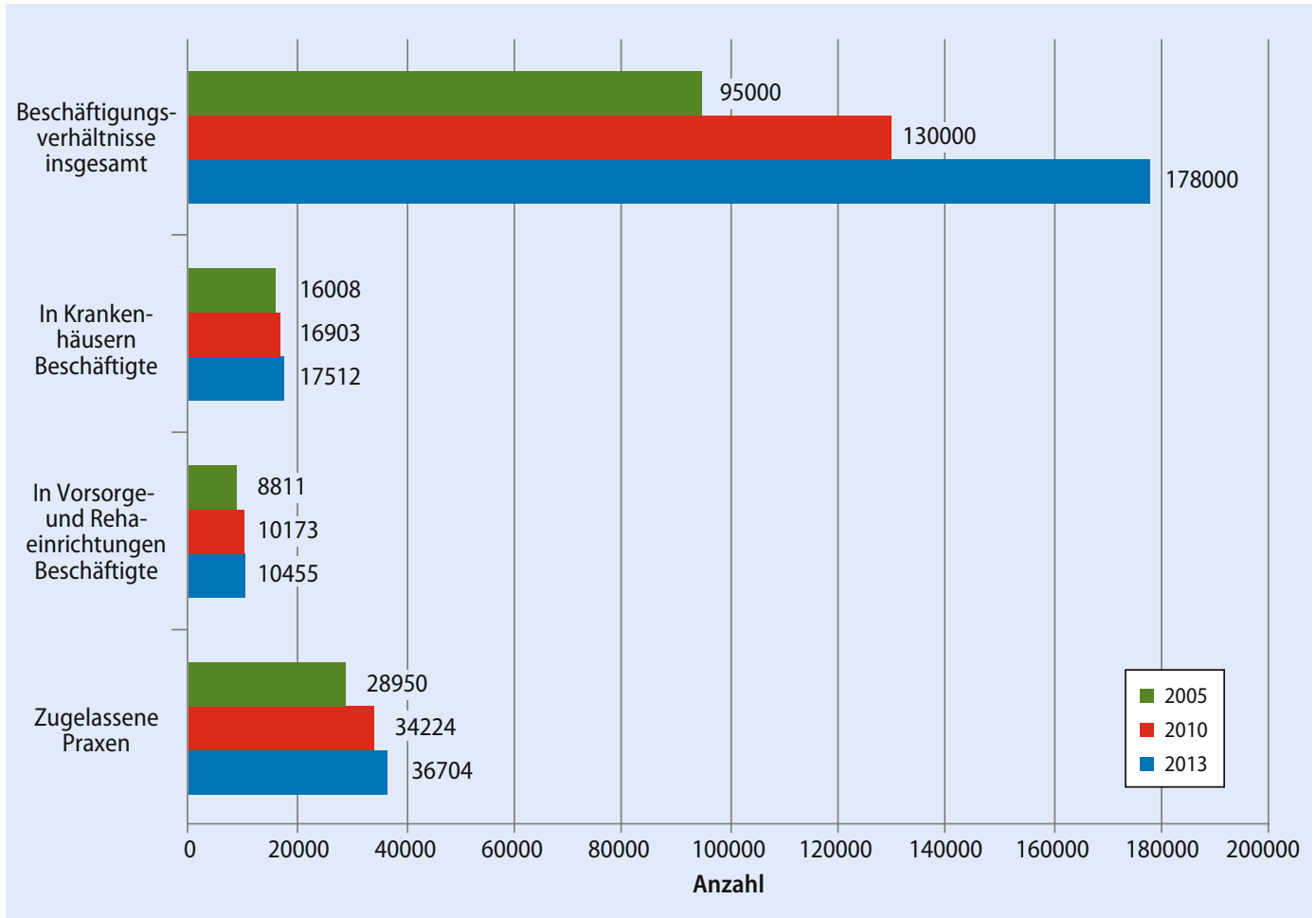

- Abb. 6.3 Physiotherapie in Deutschland: Anzahl der beschäftigten Physiotherapeuten und zugelassenen Physiotherapiepraxen

Quelle: IGES - Deutscher Verband für Physiotherapie (ZVK) e.V. (2015c)

Funktionseinschränkungen. Die ambulante Rehabilitation wird möglichst wohnortnah durchgeführt. Sie ist für Patienten ohne schwerere gesundheitliche Einschränkungen geeignet oder für Patienten, die aus familiären oder anderen Gründen, ihr häusliches Umfeld nicht länger verlassen möchten oder können. Die mobile Rehabilitation richtet sich an Patienten mit einem sehr komplexen Versorgungsbedarf und an die Patienten, die in der rehabilitativen Routineversorgung nicht ausreichend versorgt werden können. Sie ist als zusätzliche Versorgung in Einzelfällen im häuslichen Umfeld gedacht (Augurzky et al. 2011).

Je nach Stadium der Erkrankung benötigen Menschen mit MS vorübergehend oder dauerhaft pflegerische Unterstützung. Diese kann von Beratungen und Aufklärungsgesprächen zur Erkrankung über die Betreuung durch ambulante Pflegedienste bis hin zur Versorgung in teilstationären und stationären Pflegeeinrichtungen reichen (Bundesministerium für Gesundheit 2015).

\subsubsection{Physiotherapie}

Die Physiotherapie wird dem Versorgungssektor der Heilmittel zugeordnet. Ihr kommt eine erhebliche Rolle bei der Therapie von motorischen Defiziten wie z.B. Gangstörungen oder Problemen beim Greifen zu (Ziegler 2007). Sie unterstützt den Organismus bei der Neuorganisation nach Schädigung im Zentralen Nervensystem (DMSG Berlin e.V. 2015). $\mathrm{Zu}$ berücksichtigen gilt, dass die Therapie jeweils an die Alltagsverfassung des Patienten angepasst werden muss, um so seinen individuellen Bedürfnissen gerecht zu werden (DMSG Berlin e.V. 2015). Die Physiotherapie bei Patienten mit MS gilt insgesamt als komplex und lässt sich schwer vereinheitlichen (DMSG 2009). Sie kann sowohl ambulant als auch z.B. im Rahmen einer stationären Rehabilitation erfolgen (DMSG Berlin e.V. 2015), ( A Abschn. 6.5.3).

Gemäß der Vereinbarung zwischen dem Spitzenverband Bund der Krankenkassen und der Kas- 
senärztlichen Bundesvereinigung gilt die MS als Praxisbesonderheit (KVB 2013). Dies bedeutet, dass Heilmittelverordnungen, wozu die Physiotherapie zählt, den verschreibenden Arzt im Rahmen der Wirtschaftlichkeitsprüfung nicht belasten. Dies soll bewirken, dass Ärzte nicht aus monetären Aspekten, sondern patientenorientiert über die Behandlung entscheiden (KVB 2013). Die Anzahl an Physiotherapeuten in Deutschland ist in den vergangenen Jahren gestiegen ( $\bullet$ Abb. 6.3). Im Jahr 2005 waren rund 95.000 Beschäftigungsverhältnisse verzeichnet, 2013 waren es bereits 178.000 . Von diesen arbeitet knapp ein Zehntel in Krankenhäusern. Lediglich gut $5 \%$ sind in Vorsorge- oder Rehabilitationseinrichtungen beschäftigt. Die Anzahl an zugelassenen Physiotherapiepraxen stieg ebenfalls von 28.950 (2005) auf 36.704 (2013) (Deutscher Verband für Physiotherapie (ZVK) e.V. 2015c).

Speziell für MS bzw. Neurorehabilitation existieren verschiedene, uneinheitlich organisierte Fortbildungsmöglichkeiten für Physiotherapeuten in Deutschland. Beispielsweise bietet die DMSG seit 2009 eine berufsbegleitende Fortbildung "Physiotherapie bei MS« an. Die Dauer der Fortbildung beträgt rund zwölf Monate. Zugelassen werden Physiotherapeuten, welche eine zweijährige Berufserfahrung mit neurologischen Patienten, darunter mindestens zehn Patienten mit MS, nachweisen können. Jeder Kurs besteht aus maximal zwanzig Teilnehmern (DMSG 2009). Andere Fortbildungsprogramme umfassen beispielsweise nur ein Wochenende (heimerer akademie 2015) oder richten sich neben Physiotherapeuten auch an Mediziner, Ergotherapeuten und Sporttherapeuten (FobiZe 2015).

\subsubsection{Palliativversorgung}

Die Palliativversorgung ist ein elementarer und unverzichtbarer Bestandteil der Behandlung schwer betroffener Patienten mit MS. Palliativversorgung beinhaltet die interdisziplinäre Betreuung im Hinblick auf lebenserleichternde und damit die Lebensqualität erhaltende Maßnahmen wie Schmerzbehandlung, Pflege und psychotherapeutische bzw. psychologische Unterstützung.

Seit 2009 ist dieses Fachgebiet obligatorisches Lehr- und Prüfungsfach im Medizinstudium in
Deutschland und im Bereich der Ausbildung von Pflegekräften bereits seit 2003 (Busse et al. 2013). Diese Veränderung im jeweiligen Curriculum spiegelt den Bedeutungszuwachs der Palliativmedizin wider. Die Zahl der Ärzte mit Zusatzbezeichnung Palliativmedizin gemäß Weiterbildungsverordnung der Landesärztekammern stieg kontinuierlich von 2.356 Ärzten im Jahr 2009 auf 8.218 Ärzten im Jahr 2013 an. Rund die Hälfte von ihnen ist stationär tätig (Gemeinsamer Bundesausschuss 2014b).

Zentrale Akteure der Palliativversorgung sind Hospize und stationäre Palliativstationen. Während im Jahr 1996 insgesamt 28 Palliativstationen in der deutschen Krankenhauslandschaft und 30 stationäre Hospize existierten, stieg diese Zahl bis 2014 kontinuierlich auf 306 Stationen und 200 Hospizeinrichtungen an (Deutsche Gesellschaft für Palliativmedizin 2014a). Im Jahr 2008 gab es bundesweit rund 1.500 ambulante palliativmedizinische Anbieter (Deutscher Hospiz- und PalliativVerband e.V. 2015). Im Zentrum der Spezialisierten Ambulanten Palliativversorgung stehen die sogenannten Palliativ Care Teams. Die Leistungen dieser interdisziplinären Teams, bestehend aus Ärzten und Pflegekräften mit Aus- und Fortbildung im Bereich Palliativmedizin, reichen von palliativärztlicher und -pflegerischer Beratung über die Schmerz- und Symptombehandlung bis hin zur diesbezüglichen Koordination der Versorgung in Zusammenarbeit mit u. a. Hausärzten und Pflegediensten (Deutsche Gesellschaft für Palliativmedizin 2014b).

Mit dem Ziel der Aufklärung und Beratung von Patienten mit MS und deren Angehörigen zum Thema Palliativmedizin bei MS bietet das Zentrum für Palliativmedizin der Universitätsklinik Köln seit Herbst 2014 in Kooperation mit der DMSG eine kostenlose bundesweite Beratungshotline an (DMSG 2014).

\subsubsection{Pharmazeutische Unternehmen}

Als Hauptakteur in der Wirkstoffentwicklung und Arzneimittelherstellung und ebenso als forschende Einrichtungen spielen die pharmazeutischen Unternehmen in der MS-Versorgung eine wichtige Rolle. Die Arzneimitteltherapie beinhaltet die Stufentherapie ( Kap.4.1), und auch in der symptoma- 
tischen Therapie gibt es neben nicht-medikamentösen Verfahren vielfältige Indikationen für eine Arzneimitteltherapie ( $\triangleright$ Kap. 4.2).

Sechs Pharmakonzerne bezeichnen MS als einen ihrer Forschungsschwerpunkte (Verband Forschender Arzneimittelhersteller e.V. 2015). Zur etablierten medikamentösen Therapie wurden in den vergangenen Jahren folgende neue Wirkstoffe zugelassen: Fampridin, Fingolimod, Dimethylfumerat, Alemtuzumab, Teriflunomid sowie Peg-Interferon beta-1a ( Kap. 4.1).

Neben der Erforschung neuer Wirkstoffe konzentrieren sich die pharmazeutischen Unternehmen auf die Entwicklung von tablettenbasierten Darreichungsformen als Alternative zur Injektion. Studien haben gezeigt, dass Faktoren im Zusammenhang mit Injektionstherapien bei MS, wie Hautreaktionen und Schmerzen, die Therapietreue maßgeblich beeinflussen können (Patti 2010; Remington et al. 2013). Von den in den letzten Jahren entwickelten Wirkstoffen wurden Fingolimod, Dimethylfumarat und Teriflunomid als Tabletten bzw. Kapseln zur Behandlung der MS in der EU zugelassen. Ergänzend zur Arzneimittelentwicklung und -herstellung unterstützen pharmazeutische Unternehmen finanziell die Arbeit von Patientenorganisationen und finanzieren ebenso Forschungsprojekte weiterer externer Einrichtungen. So nimmt die DMSG von pharmazeutischen Unternehmen finanzielle Unterstützung unter der Voraussetzung entgegen, dass eine finanzielle und inhaltliche Unabhängigkeit der Arbeit der DMSG gewährleistet ist (DMSG 2000).

\subsubsection{Patientinnen und Patienten}

Die Patientenorientierung ist ein zentrales Grundelement bei der Untersuchung der medizinischen Versorgungssituation (Pfaff et al. 2011). Trotz der therapeutischen Fortschritte bleibt MS eine bisher nicht heilbare Erkrankung, die zu Beeinträchtigungen und zum Verlust von körperlichen und kognitiven Funktionen führt. Individuelle Krankheitsverläufe sowie verschiedene Ressourcen und Bedürfnisse zur Teilhabe gestalten die patientenorientierte Behandlung komplex. Zur Versorgungsoptimierung ist eine Berücksichtigung individueller Bedürfnisse wichtig. Aus Perspektive Betroffener wird ein Großteil der »unmet needs « im Bereich der gesundheitlichen Versorgung verortet. Potenziale sahen die Patientinnen und Patienten vor allem bezüglich des Zugangs zu gesundheitlichen Diensten (z. B. eine geringere Distanz zu Fachärzten), der Arzt-Patienten-Interaktion (mehr Partizipation bei Behandlungsentscheidungen) sowie in einer gesteigerten Kontinuität beim Wechsel zwischen verschiedenen Versorgungssektoren, wie eine Befragung von Patientinnen und Patienten mit MS ergab (Galushko et al. 2014). So wurden u. a. »Brüche« zwischen der ambulanten und stationären Versorgung bemängelt. Hinzu kamen Informationsbedarfe der Patientinnen und Patienten im Hinblick auf Behandlungsoptionen und dem Verlauf der Erkrankung. Weiterhin wurde der Wunsch nach Aufrechterhaltung der biografischen Kontinuität im Berufs- und Privatleben und damit auch der Aufrechterhaltung der eigenen Identität geäußert (Galushko et al. 2014).

Das Leit- und Idealbild für die Berücksichtigung der Patientenbedarfe im Rahmen der medizinischen Versorgung ist das partnerschaftlichen Modells der Arzt-Patienten-Kommunikation, welches durch einen reziproken Austausch über Werte und Ziele gekennzeichnet ist. Wesentliche Konzepte dieser Arzt-Patienten-Beziehung sind die partizipative Entscheidungsfindung (»shared-decision-making ") und Empowerment. Die partizipative Entscheidungsfindung zielt durch einen aktiven Informationsaustausch sowie Aushandlungsprozesse auf eine gemeinsame Entscheidungsfindung ab, für die sowohl der Arzt als auch die Patientin und der Patient die Verantwortung übernehmen, und soll die Betroffenen in die Lage versetzen, informiert an medizinischen Entscheidungen teilzunehmen (Empowerment). Wenn die Patientin und der Patient Entscheidungsträger ihrer eigenen Behandlung sind, kann von einer deutlich höheren Adhärenz ausgegangen werden (Faller 2012).

Versorgungsnetzwerke wie »AmbulanzPartner « unterstützen Patientinnen und Patienten in der Organisation ihrer Behandlung. Sie erleichtern beispielsweise die Suche nach geeigneten Therapeuten, Hilfsmittelexperten oder Apotheken sowie deren Abstimmung aufeinander. Weiterhin können sie bei der Rezeptabwicklung helfen und durch Bewertungsportale Transparenz bezüglich der Qualität von Therapien oder Anbietern gesundheitsbezoge- 
ner Leistungen schaffen (AmbulanzPartner 2015). Kooperationen wie das Portal der "weißen Liste" und der Krankenkassen sowie weitere Portale unterstützen Patientinnen und Patienten sowie Angehörige beispielsweise bei der Auswahl von Krankenhäusern, Ärzten oder Pflegeeinrichtungen durch ein umfassendes Bewertungssystem (Rupprecht und Schulte 2013).

\subsection{Modellprojekt: Integrierte Versorgung Multiple Sklerose am Beispiel der Region Nordrhein}

Seit 2003 können gemäß $\$ 140$ SGB V Modelle der IV umgesetzt werden. Dies ermöglicht Krankenkassen, Verträge über eine verschiedene Leistungssektoren übergreifende Versorgung oder eine multidisziplinär-fachübergreifende Versorgung mit definierten Vertragspartnern abzuschließen ${ }^{12}$. Diese Vertragspartner sind in $\$ 140$ b Abs. 1 definiert und umfassen u. a. einzelne niedergelassene Ärzte, Träger zugelassener Krankenhäuser, Träger von stationären Vorsorge- und Rehabilitationseinrichtungen, Träger von ambulanten Rehabilitationseinrichtungen, Pflegekassen und Pflegeeinrichtungen, Praxiskliniken, pharmazeutische Unternehmen und Hersteller von Medizinprodukten. Die Teilnahme der Versicherten an IV-Modellen ist freiwillig. Zudem haben die Versicherten das Recht, umfassend über die Verträge der IV, die teilnehmenden Leistungserbringer, die Leistungen und über die vereinbarten Qualitätsstandards informiert zu werden ${ }^{13}$.

Zu den Zielen der IV gehören (Nelles et al. 2010; Wirtz 2010):

- die Optimierung der qualitätsgesicherten Versorgung der Patienten,

- die schnelle und präzise Feststellung der Indikation,

- die Verbesserung der Schnittstellen für eine sektorenübergreifende Versorgung (ambulanter und stationärer Sektor),

- eine leitliniengerechte und systematische Koordination der Behandlung,
- die Verbesserung des Krankheitsverlaufs und der Lebensqualität der Patienten,

- die Reduktion von stationären Aufenthalten,

- ein zielgenauer Einsatz von Mitteln und Ressourcen sowie

- eine bevölkerungsbezogene, flächendeckende Versorgung.

Im Rahmen von IV-Modellen sind umfassende, standardisierte Datenerhebungen, anhand derer differenzierte Patientenverläufe analysiert und verglichen werden können, möglich. Der potenzielle Nutzen des IV-Modells für Patienten kann sich u. a. aus dem dichten Netz ausgewiesener Experten, einer schnelleren Diagnosesicherung sowie dem Schutz vor nicht wirksamen Behandlungen und deren möglichen Nebenwirkungen ergeben. Eine individuelle Fallbetreuung durch sogenannte »CaseManager « bietet einen direkten Ansprechpartner, der als Lotse für den Patienten durch das komplexe Behandlungsgeschehen fungieren kann.

Im IV-Modell der Region Nordrhein werden modulare Patientenschulungsprogramme, die patientenindividuell modifizierbar sind und $\mathrm{u}$. a. die Module Krankheitsbewältigung, Fatigue-Management und Stressbewältigung umfassen, angeboten. Diese Problembereiche werden im Rahmen des GKV-Leistungskataloges nur unzureichend berücksichtigt (Landeszentrum Gesundheit Nordrhein-Westfalen 2015).

Als Vorteile auf Seiten der beteiligten Krankenkassen ist die qualitätsgesicherte Behandlung, die die Krankheitsentwicklung und Begleiterkrankungen günstig beeinflussen kann, zu sehen. Dies kann auch zu einer höheren Lebensqualität und einer längeren Aufrechterhaltung der Berufsfähigkeit führen. Der zielgenauere Mitteleinsatz kann sich auch an anderen Stellen kostenreduzierend auswirken, zum Beispiel durch Vermeidung unnötiger Arztbesuche und Doppeluntersuchungen sowie Verringerung stationärer Aufenthalte durch die Ausweitung ambulanter Maßnahmen. Im Vordergrund der IV-Modelle steht die Bündelung der Versorgungskompetenzen (Limmroth et al. 2006). Innerhalb der Versorgung sind dabei auch die besonderen Anforderungen und Bedarfe an die geschlechtsspezifische Versorgung zu berücksichtigen, denn Frauen sind deutlich häufiger betroffen als Männer ( $\triangleright$ Kap. 2). 
Voraussetzungen für eine Teilnahme an IVModellen zur MS auf Patientenseite sind eine gesicherte Diagnose, eine Einverständniserklärung sowie die Zugehörigkeit zu einer der teilnehmenden Krankenkassen. Niedergelassene Neurologen müssen für eine Teilnahme folgende Qualifikationen erfüllen: mindestens fünf Jahre Erfahrung in der Behandlung von Patienten mit MS, mindestens 25 Patienten mit MS pro Jahr, Bereitschaft zur standardisierten Dokumentation, Möglichkeit der Liquordiagnostik, Zusammenarbeit mit Neuroradiologen und Urologen, Bereitschaft zur ambulanten Schubtherapie und Durchführung von Schulungsmaßnahmen. Für teilnehmende Kliniken ergeben sich zusätzlich folgende Voraussetzungen: Behandlung von mindestens 100 Patienten mit MS pro Jahr, Möglichkeit einer an der Krankheitsaktivität orientierten Arzneimitteltherapie sowie die Gewährleistung und Sicherstellung der ambulanten Weiterversorgung der Patienten innerhalb des IV-Modellrahmens mit Weitergabe der relevanten Behandlungsinformationen. Des Weiteren müssen definierte Ansprechpartner und Modalitäten für eine stationäre Aufnahme gegeben sein (Limmroth et al. 2006; Wirtz 2010).

Im Jahr 2003 wurde von der DGN in Kooperation mit dem BDN ein erstes Rahmenkonzept für die IV von Patienten mit MS entwickelt. In Anlehnung an dieses Konzept wurde im Jahr 2006 in Zusammenarbeit mit der AOK Rheinland/Hamburg und dem Landesverband der DMSG ein IV-Vertrag für die Region Rheinland (Versorgungsbereich der Ärztekammer Nordrhein, NRW) geschlossen (Limmroth et al. 2006). Das Leistungsspektrum sowie die Vergütung des IVMS-Versorgungsvertrages Rheinland wurden zwischen den Kostenträgern, den beteiligten Neurologen, dem BDN und der DMSG ausgehandelt. Zentrale Steuerungsinstanz des IV-Netzwerkes ist der Netz-Beirat, der u. a. Vertragsfragen und Meinungsverschiedenheiten klärt. Er setzt sich aus drei Vertretern der Leistungserbringer und drei Vertretern der Kostenträger zusammen. Die spezifischen Vertragsleistungen des IV-Angebots sind in Tabelle 6.2 dargestellt. Alle Leistungen können mit Ausnahme der Behandlungspauschale im stationären oder ambulanten Bereich erbracht werden. In den Kliniken erfolgt die Vergütung der Behandlungspauschalen über DRG (Diagnosis Related Groups). Gesetzliche Regelleis- tungen werden entsprechend den üblichen Regelungen (Einheitlicher Bewertungsmaßstab, DRG) vergütet (Limmroth et al. 2006; Nelles et al. 2010). Im Vergleich zu IV-Verträgen anderer Regionen (z. B. Hessen) wurde im hier beschriebenen IVMSVersorgungsvertrag Rheinland kein Medikamentenbudget vordefiniert (Limmroth et al. 2006). Für die Leistungserbringung wurden spezifische Behandlungspfade entsprechend den Leitlinien der DGN definiert, die für alle Teilnehmer des IV-Projektes bindend sind. Hierbei wurde u. a. zwischen planbaren und unvorhersehbaren Leistungen differenziert. Planbare Leistungen sind u. a. die in - Tab. 6.1 dargestellten Jahres- und Quartalskonsultationen. Da bei Patienten mit MS typischerweise Komplikationen auftreten, die Behandlungen außerhalb planbarer Konsultationen bedürfen, wurden in einem IV-Behandlungspfad zusätzlich vier Gruppen von unvorhersehbaren Krankheitskomplikationen aufgenommen, deren Berücksichtigung in der Regelversorgung nur begrenzt möglich ist. Hierzu gehören Therapiekomplikationen, neuropsychologische Störungen, psychosoziale Probleme und Funktionsverschlechterungen (Nelles et al. 2010).

Gegenwärtig sind mehr als 120 niedergelassene Neurologen, 14 Kliniken und Rehabilitationseinrichtungen an dem IVMS-Versorgungsvertrag Rheinland beteiligt; etwa 2.000 Patienten mit MS sind eingeschrieben. Zu den teilnehmenden Krankenkassen gehörten im Jahr 2015 die AOK Rheinland/Hamburg, die IKK Nordrhein, Knappschaft, die KKH-Allianz, sowie die Landwirtschaftliche und Gärtner-Krankenkasse (Kliniken der Stadt Köln gGmbH 2015).

Empirische Evaluationen zur Qualität der IV liegen zurzeit nur in begrenztem Umfang vor, sind allerdings für die Beurteilung von Versorgungsstrukturen von großer Bedeutung (Nelles et al. 2010). Nelles et al. (2010) haben daher eine zweijährige, prospektive Verlaufsbeobachtungsstudie durchgeführt, um das Ausmaß der Zielerreichung des vorgestellten IV-Modellprojektes zu überprüfen.

In der Beobachtungsstudie konnten die Basisdatensätze von 1.582 Patienten ausgewertet werden. Für die Verlaufskontrolle nach zwei Jahren standen Daten von 319 Patienten zur Verfügung. Zur Baseline betrug der Altersdurchschnitt 49 Jahre (SD = 14 Jahre). Frauen machten den überwiegenden Anteil 
- Tab. 6.1 Leistungen der Integrierten Versorgung MS - Modellregion Nordrhein

\begin{tabular}{|c|c|c|}
\hline Leistung & Beschreibung & Frequenz (pro Jahr) \\
\hline $\begin{array}{l}\text { Eingangsuntersuchung; } \\
\text { Jahreskonsultation }\end{array}$ & $\begin{array}{l}\text { Anamnese der klinischen Gesamtsituation bei Aufnah- } \\
\text { me, Erhebung mittels der Expanded Disability Status } \\
\text { Scale, defizitorientierte Therapieplanung }\end{array}$ & 1 \\
\hline Quartalskonsultation & $\begin{array}{l}\text { Verlaufskontrolle, Reha-Assessment, defizitorientierte } \\
\text { Therapieplanung, Koordination der Heilmittelerbrin- } \\
\text { gung, ggf. Stabilisierung des stationären Behandlungs- } \\
\text { erfolgs }\end{array}$ & 3 \\
\hline Dokumentation & SF-54; Minimal Basis Dataset & $\begin{array}{l}\text { 1-mal: SF } 54 \\
\text { 1-mal: Minimal Basis Dataset }\end{array}$ \\
\hline Überleitungspauschale & $\begin{array}{l}\text { Aufnahme und Entlassungskoordination mit Kopie aller } \\
\text { Befunde zur Absicherung der Kontinuität }\end{array}$ & 2 \\
\hline Behandlungspauschale & $\begin{array}{l}\text { Ambulante Schub- und Eskalationsbehandlung, } \\
\text { therapiebegleitendes Monitoring, Abklärung von } \\
\text { Komplikationen }\end{array}$ & 4 \\
\hline Patientenschulung & Konzipiert durch die DMSG & 1 \\
\hline Fallkonferenzen & $\begin{array}{l}\text { Abstimmung mit stationären Behandlern und Heil- } \\
\text { mittelerbringern }\end{array}$ & 2 \\
\hline
\end{tabular}

der Teilnehmenden aus (72,5\%). Im Durchschnitt lag die Erkrankungsdauer bei 13,1 Jahren (SD = 9,1 Jahre). Die meisten Patienten (80,1 \%) wiesen einen primär schubförmigen Verlauf auf, während bei $15,8 \%$ ein primär progredienter Verlauf mit und ohne Schübe vorlag. Hinsichtlich der eher qualitativen Indikatoren des IV-Modells zeigte sich, dass in der Population der IV-Patienten nach zwei Jahren die Zahl der stationären Akutbehandlungen von $25,7 \%$ auf 9,1 \% im Vergleich zur Baseline signifikant abnahm. Bei Patienten, die eine Erkrankungsdauer von weniger als fünf Jahren aufwiesen, zeigte sich in dem gleichen Studienzeitraum ebenfalls eine signifikante Reduktion akutstationärer Behandlungen von $58,3 \%$ auf $13,2 \%$. In Hinblick auf den Behinderungsgrad zeigte sich keine signifikante Veränderung nach zwei Jahren im Vergleich zur Basisdokumentation. Zur Baseline lag der durchschnittliche Wert auf der Expanded Disability Status Scale (EDSS) bei 3,9 (SD = 3,3), nach zwei Jahren bei 4,0 $(\mathrm{SD}=4,1)$. In Bezug auf die gesundheitliche Lebensqualität, operationalisiert mit dem MSQoL-54 (Multiple Sclerosis Quality of Life-Erhebungsbo- gen), zeigten sich keine statistisch signifikanten Veränderungen zwei Jahre nach Beginn des IV-Modellprojektes (Nelles et al. 2010).

Insgesamt lässt sich festhalten, dass in Korrespondenz mit den inhaltlichen Zielen des IVMSProjektes Rheinland eine Reduktion der akutstationären Behandlungsaufenthalte beobachtet werden konnte. Als Grund für die Abnahme der akutstationären Behandlungen wird von den Autoren v. a. die im IV-Vertrag festgelegte ambulante Behandlung von MS-Schüben genannt, da Krankheitsschübe bei Patienten mit MS eine wesentliche Ursache für stationäre Aufnahmen darstellen. $\mathrm{Ob}$ und wann eine ambulante oder stationäre Schubbehandlung indiziert ist, wird in den Behandlungspfaden des IVVertrages beschrieben. Als weitere Ursache für die beobachtete Abnahme stationärer Behandlungen werden die von der DMSG entwickelten Patientenschulungsprogramme genannt, deren Ziel die Befähigung der Patienten zu kompetenten und aktiven Behandlungsentscheidungen ist. Der durch MSbedingte Behinderungsgrad hat sich nicht signifikant verändert, was angesichts des Studienzeit- 
raums von zwei Jahren einem Verlauf unter krankheitsmodifizierenden Medikamenten nahe kommt (Nelles et al. 2010). Die gesundheitsbezogene Lebensqualität (bestehend aus psychischen und körperlichen Komponenten) zeigte im Allgemeinen ebenfalls keine signifikante Veränderung in der Verlaufsbeobachtung. Allerdings hatten Patienten mit eingeschränkter Gehfähigkeit eine insgesamt niedrigere Lebensqualität im Vergleich zu Patienten mit höherer Mobilität (Nelles et al. 2010).

Bei Betrachtung der Studienergebnisse sollte berücksichtigt werden, dass aufgrund des Studiendesigns keine kausalen Schlussfolgerungen zulässig sind; die beobachtete Verringerung der stationären Behandlungsaufenthalte kann auch auf andere als von den Autoren genannte Ursachen zurückzuführen sein. Ein späterer Kontrollgruppenvergleich von Nelles et al. zeigte einen ähnlichen Effekt bezüglich der Abnahme von akutstationären Behandlungen. Demnach haben Patienten, die an dem IVMS-Projekt Rheinland teilnahmen, etwa $50 \%$ weniger akutstationäre Behandlungen in Anspruch genommen und eine geringere Komplikationsrate im Vergleich zu Patienten mit MS, die ausschließlich Regelleistungen erhielten. Da für die Kontrollgruppe keine Daten zum MS-spezifischen Behinderungsgrad und zur gesundheitsbezogenen Lebensqualität vorlagen, war diesbezüglich ein Vergleich zwischen den Teilnehmern des IV-Modells und Patienten aus der Regelversorgung nicht möglich. Um vergleichbare Gruppen zwischen IV und Regelversorgung zu bilden wurde für das Matching das PropensityScore Verfahren angewendet. Dabei wurden u. a. Merkmale wie Alter und Geschlecht, Diagnosen sowie Anzahl der stationären Aufenthalten, psychoorganische Störungen, Inanspruchnahme spezifischer Hilfsmittel, Schubtherapie, Eskalationstherapie, behandelte Spastik und Therapie mit Antidepressiva jeweils im Vorjahr herangezogen. In der Verlaufsbeobachtung über vier Jahre ist sowohl der Schweregrad der Behinderung (EDSS) als auch die Lebensqualität weitgehend gleich geblieben. Eine Konstanz in der Lebensqualität und der Gehfähigkeit bei Patienten mit MS ist im Verlauf tendenziell positiv zu bewerten (Nelles et al. 2013).

Betrachtet man die vorangegangene Studie aus dem Jahr 2010, lässt sich anhand der Untersuchungsergebnisse der Kontrollvergleichsstudie aus dem Jahr 2013 eine empirisch stärker fundierte Aussage bezüglich der Qualität des IV-Modells ableiten. Wie von den Autoren (Nelles et al. 2013, 2010) diskutiert, sollten in Zukunft auch differenzierte Kostenanalysen sowie prospektive Verlaufsuntersuchungen bezüglich der Lebensqualität folgen. Des Weiteren ist anzumerken, dass in beiden von Nelles et al. durchgeführten Untersuchungen (Nelles et al. 2013, 2010) im Wesentlichen zwei Messzeitpunkte (Baseline und zwei Jahre später) miteinander verglichen wurden, die die Abbildung eines Verlaufs folglich nicht zulassen. So können z. B. mögliche Fluktuationen in der Lebensqualität, die sich innerhalb dieses Zeitraums ergeben haben, nicht nachvollzogen werden. Für die weitere Qualitätssicherung wäre daher eine höhere Dichte von Untersuchungszeitpunkten durchaus wünschenswert.

Zukünftige Ziele des IV-Modells in der Region Nordrhein bestehen in der Aufnahme neuer Immuntherapien und in der Implementierung von Algorithmen zur Patientensicherheit, um unerwünschte Ereignisse frühzeitig erkennen zu können.

\subsection{Forschung}

Um die Versorgung eines Patienten optimal gestalten zu können, ist Versorgungsforschung nötig, da sie es ermöglicht, Mängel aufzudecken und diese entsprechend zu beseitigen. Versorgungsforschung ist definiert als »fachübergreifendes Forschungsgebiet, das die Kranken- und Gesundheitsversorgung und ihre Rahmenbedingungen beschreibt und kausal erklärt, zur Entwicklung wissenschaftlicher fundierter Versorgungskonzepte beiträgt, die Umsetzung neuer Versorgungskonzepte begleitend erforscht und die Wirksamkeit von Versorgungsstrukturen und-prozessen unter Alltagsbedingungen evaluiert« (Pfaff 2003). Sie fußt dabei auf den Grundkonzepten der Ergebnisorientierung, der Multidisziplinarität bzw. Multiprofessionalität und der Patientenorientierung (Pfaff et al. 2011).

Bereits aus der Definition geht hervor, dass der Versorgungsforschung zahlreiche Forschungsbereiche vorangestellt sind, wie beispielsweise die medizinische Grundlagenforschung oder Forschung im Bereich der Arzneimitteltherapie. In Deutschland erforschen zahlreiche wissenschaftliche Institutionen 
die Erkrankung MS aus unterschiedlichen Blickwinkeln und befassen sich mit etablierten und potenziellen medikamentösen und nicht-medikamentösen Therapiewegen. Zentrale Akteure in Deutschland sind hierbei universitäre und private Forschungsinstitute, die medizinischen Fachgesellschaften und die forschende Pharmaindustrie ( Abschn. 6.5.6).

Das vom Bundesministerium für Bildung und Forschung seit 2009 geförderte Kompetenznetzwerk MS (KKNMS) zielt auf die Förderung und Vernetzung der MS-bezogenen nationalen und auch internationalen Forschungsaktivitäten. Es ist zusammen mit der DGN Herausgeber der Leitlinie zur Diagnostik und Therapie der MS (DGN 2014). Ein aktuelles Forschungsprojekt des KKNMS befasst sich mit der Gewinnung von Erkenntnissen zu Biomarkern, um diagnostische und therapeutische Ansätze zu optimieren. Diese MS-Kohortenstudie mit einer geplanten Laufzeit von zehn Jahren setzt sich aus 1.000 Patienten mit klinisch-isoliertem Syndrom (KIS) bzw. einem Frühstadium der MS zusammen. Am Projekt beteiligt sind klinische Zentren und weitere medizinische Einrichtungen. Die Projektleitung obliegt der Neurologischen Klinik der Universität Bochum (Kompetenznetz Multiple Sklerose 2015d). Bei dem seit 2013 bestehenden REGIMS-Register handelt es sich um ein Immuntherapieregister zur Erfassung, Systematisierung und Untersuchung von mit MS-Medikamenten assoziierten Nebenwirkungen. Das Projekt wird vom Institut für Epidemiologie und Sozialmedizin der Universität Münster geleitet. Die Zusammenarbeit erfolgt unter anderen mit dem BfArM sowie mit dem Paul-Ehrlich-Institut (Kompetenznetz Multiple Sklerose 2015c). Die KKNMS führt zudem eine Biobank mit Gewebe des Zentralnervensystems sowie nicht-zellulären und zellulären Proben. Zukünftig geplant ist die projektgebundene Bereitstellung der Bioproben für Mitglieder des KKNMS sowie externe Wissenschaftler mittels Antragstellung. Hierfür hat das KKNMS einen eigenen Fachausschuss eingerichtet (Kompetenznetz Multiple Sklerose 2015b). Im Bereich Versorgungsforschung untersucht das KKNMS mit Leitung der Universität Hamburg, wie Patientenwissen zur Immuntherapie bei MS die Wahl der Behandlungsart und die Compliance des Patienten prägt. Diese sogenannte DECIMS (Decision Coaching in Multiple Sclerosis) erfolgt unter Mitwirkung von mehreren MS-Zentren und beinhaltet unter anderen die Entwicklung von Patienteninformationssystemen (Kompetenznetz Multiple Sklerose 2015a). Unter Leitung der Georg-August-Universität Göttingen baut das KKNMS ein ausschließlich auf Kinder bezogenes Patientenregister namens CHILDREN MS auf, welches Erkenntnisse zur Epidemiologie der MS bei jüngeren Altersgruppen liefern soll.

Das Institut für MS-Forschung (IMSF) der Universität Göttingen beschäftigt sich seit Institutsgründung im Jahr 2004 primär aus neuroimmunologischer Perspektive mit der Erkrankung MS, um neue Therapieoptionen zu generieren und Wirkprozesse bestehender Behandlungen besser nachvollziehen zu können. Die Gründung des IMSF erfolgte mit Unterstützung der Gemeinnützigen Hertie Stiftung (Universitätsmedizin Göttingen).

Anhand der Vielzahl der Projekte lässt sich erkennen, dass in Bezug auf das Krankheitsbild, die Diagnose und Therapieverfahren der MS weiterhin Forschungsbedarf besteht. Gemäß der Definition von Versorgungsforschung sind jedoch auch die Kenntnisse anderer Faktoren von entscheidender Bedeutung. So müssen Informationen über den $\mathrm{Pa}$ tienten gesammelt und seine Bedarfe und Nachfrage sowie dessen Präferenzen ermittelt werden, um eine patientenorientierte Versorgung zu ermöglichen. Aus volkswirtschaftlicher Perspektive sind zudem Kenntnisse über die sozioökonomischen Auswirkungen einer Krankheit und deren Therapieformen relevant (Pfaff et al. 2011).

Erkenntnisse, die im Rahmen kontrollierter klinischer Studien erzielt werden, können nur mit gewissen Einschränkungen auf die alltägliche Routinebehandlung übertragen werden, da sich die Patienten mit MS, die innerhalb der Routineversorgung einen Arzt aufsuchen, von denen unterscheiden, die an einer klinischen Studie teilnehmen. Beispielhaft sei hier "PANGEA « als eine unter Alltagsbedingungen durchgeführte Studie genannt. Aus den Daten von 4.000 Patienten mit RRMS sollen Rückschlüsse über die Sicherheit und die Effektivität des Arzneistoffs Fingolimod gezogen werden. Zudem wird eine Subpopulation von 800 Patienten hinsichtlich Parametern wie gesundheitsbezogene Lebensqualität, Therapiezufriedenheit und Compliance untersucht (Ziemssen et al. 2015). Die »Pro- 
spective pharmacoeconomic cohort evaluation (PEARL) Study“ gibt beispielsweise Hinweise auf die Versorgungsituation unter Alltagsbedingungen von Patienten mit RRMS ( $\mathrm{n}=1705)$. Untersucht werden unter anderem sowohl aus Patientenperspektive als auch aus medizinischer Sicht der Gesundheitsstatus und Krankheitsverlauf sowie die Therapiezufriedenheit bzw. -wirksamkeit. Weiterhin wird die (Arbeits-) Produktivität betrachtet. Erste Ergebnisse liegen als Kongressbeiträge vor (Vormfelde u. Ziemssen 2015a,b,c).

Das Multiple Sklerose und Kinderwunsch-Register (DMSKW) erfasst prospektiv Schwangerschaften oder Kinderwunschbehandlungen von
Patientinnen mit MS. Projektziel ist die Erhebung von Daten hinsichtlich des Verlaufs der MS, des Einflusses immunmodulatorischer Therapien auf die Schwangerschaft sowie Risiko- und Resilienzfaktoren für Schübe nach der Geburt. Bisher wurden knapp 400 Schwangerschaften in der Datenbank dokumentiert (Stand 22.01.2016). Teilnahmevoraussetzung ist eine bereits bestehende Schwangerschaft, ein aktueller Kinderwunsch oder ein unerfüllter Kinderwunsch von Frauen mit MS, die eine reproduktionsmedizinische Behandlung planen. Das DMSKW wird von forschenden Pharmaunternehmen unterstützt (DMSKW 2015).

Open Access Dieses Kapitel wird unter der Creative Commons Namensnennung-Nicht kommerziell 4.0 International Lizenz (http://creativecommons.org/licenses/by-nc/4.0/deed.de) veröffentlicht, welche für nicht kommerzielle Zwecke die Nutzung, Vervielfältigung, Bearbeitung, Verbreitung und Wiedergabe in jeglichem Medium und Format erlaubt, sofern Sie den/die ursprünglichen Autor(en) und die Quelle ordnungsgemäß nennen, ein Link zur Creative Commons Lizenz beifügen und angeben, ob Änderungen vorgenommen wurden.

Etwaige Abbildungen oder sonstiges Drittmaterial unterliegen ebenfalls der genannten Creative Commons Lizenz, sofern sich aus der Abbildungslegende oder der Quellreferenz nichts anderes ergibt. Sofern solches Drittmaterial nicht unter der genannten Creative Commons Lizenz steht, ist eine Vervielfältigung, Bearbeitung oder öffentliche Wiedergabe nur mit vorheriger Zustimmung des betreffenden Rechteinhabers oder auf der Grundlage einschlägiger gesetzlicher Erlaubnisvorschriften zulässig.

\section{Literatur}

Amelung VE, Bergmann F, Hauth I, Jaleel E, Meier U, Reichmann H, Roth-Sackenheim C, (Hrsg,), (2010) Innovative Konzepte im Versorgungsmanagement von ZNS-Patienten. Berlin: MWV Medizinisch Wissenschaftliche Verlagsgesellschaft. ISBN: 978-3-941468-18-4.

Anders D, Oschmann P (2006) Von der Versorgungsforschung zur Integrierten Versorgung - Am Beispiel der Multiplen Sklerose. Spiegel der Forschung 23(1/2), 68-75.

Augurzky B, Reichert A, Scheuer M (2011) Faktenbuch Medizinische Rehabilitation 2011. RWI Materialien. Essen: Rheinisch-Westfälisches Institut für Wirtschaftsforschung. ISBN: 978-3-86788-285-9.

BARMER GEK NRW, DAK-Gesundheit, Berufsverband Deutscher Nervenärzte (Landesverband Westfalen) u. Kassenärztliche Vereinigung Westfalen-Lippe (2014) Gemeinsame Presseinformation vom 24.07.2014. Neuer Vertrag in Westfalen-Lippe seit 1. Juli 2014: VersorgungsAssistentinnen entlasten Nervenärzte. Düsseldorf, Dortmund, Frankfurt. https://www.kvwl.de/presse/pm/2014/ pdf/2014_07_24_1.pdf [Abruf am: 30. April 2015].
BDN (2015a) Berufsverband Deutscher Neurologen. http:// www.bv-neurologe.de/themen-bdn/fortbildungsakademie-bdn.html [Abruf am: 28. April 2015].

BDN (2015b) Fortbildungsakademie. Berufsverband Deutscher Neurologen. http://www.bv-neurologe.de/ themen-bdn/fortbildungsakademie-bdn.html [Abruf am: 11. Juni 2015].

BDN (2015c) Rehabilitation. Berufsverband Deutscher Neurologen. http://www.bv-neurologe.de/themen-bdn/rehabilitation-bdn.html [Abruf am: 11. Juni 2015].

BDNR (2015) Aufnahmeantrag für den Berufsverband Deutscher Neuroradiologen e.V. http://www.bdnr.de/ bilder/Aufnahmeantrag.pdf [Abruf am: 11. Juni 2015].

BNR (2015) Bundesverband NeuroRehabilitation e.V. http://www.bv-neuroreha.de/ [Abruf am: 28. April 2015].

Bundesärztekammer (2007) Abbildungen und Tabellen zur Ärztestatistik 2006. http://www.bundesaerztekammer. de/fileadmin/user_upload/downloads/Aerztestatistik 2006.pdf [Abruf am: 15. Dezember 2015].

Bundesärztekammer (2011) Abbildungen und Tabellen zur Ärztestatistik 2010. http://www.bundesaerztekammer. 
de/fileadmin/user_upload/specialdownloads/Stat10 Tab03.pdf [Abruf am: 15. Dezember 2015].

Bundesärztekammer (2013) (Muster-)Weiterbildungsordnung 2003 in der Fassung vom 28.06.2013. http://www.bundesaerztekammer.de/downloads/20130628-MWBO_ V6.pdf [Abruf am: 18. Dezember 2014].

Bundesärztekammer (2014) Abbildungen und Tabellen zur Ärztestatistik 2013. http://www.bundesaerztekammer. de/fileadmin/user_upload/downloads/Stat13AbbTab.pdf [Abruf am: 23. April 2015].

Bundesärztekammer (2015) Abbildungen und Tabellen zur Ärztestatistik 2014. http://www.bundesaerztekammer. de/fileadmin/user_upload/downloads/pdf-Ordner/ Statistik2014/Stat14Tab03.pdf [Abruf am: 15. Dezember 2015].

Bundesärztekammer (2015) (Muster-)Berufsordnung für die in Deutschland tätigen Ärztinnen und Ärzte- MBO-Ä 1997 - in der Fassung des Beschlusses des 118. Deutschen Ärztetages 2015. Frankfurt am Main: Deutsches Ärzteblatt. DOI: 10.3238/arztebl.2015.mbo_daet2015.

Bundesministerium für Gesundheit (2015) Zahlen und Fakten zur Pflegeversicherung. Stand: 13.03.2015. http://www. bmg.bund.de/fileadmin/dateien/Downloads/Statistiken/ Pflegeversicherung/Zahlen_und_Fakten/150420_Zahlen_und_Fakten_Pflegeversicherung_03-2015.pdf [Abruf am: 23. April 2015].

Bundespsychotherapeutenkammer (2013) EBM Ziffern für neuropsychologische Therapie beschlossen. http://www. bptk.de/aktuell/einzelseite/artikel/ebm-ziffern.html [Abruf am: 20. Oktober 2015].

Bundeszentrale für politische Bildung (2012) Staatliche Akteure. http://www.bpb.de/politik/innenpolitik/gesundheitspolitik/72726/staatliche-akteure [Abruf am: 28. April 2015].

Busse R, Blümel M, Ognyanova D (2013) Das deutsche Gesundheitssystem. Akteure, Daten, Analysen. Berlin: MWV Medizinisch Wissenschaftliche Verlagsgesellschaft.

Busse R, Panteli D, Henschke C (2015) Arzneimittelversorgung in der GKV und 15 anderen europäischen Gesundheitssystemen: Ein systematischer Vergleich. Working papers in health policy and management, Band 11. Berlin: Universitätsverlag der TU Berlin. [Abruf am: 19.10.2015].

BVDN (2015a) Berufsverband Deutscher Nervenärzte. http:// www.bvdn.de/ [Abruf am: 11. Juni 2015].

BVDN (2015b) Spitzenverband ZNS. Berufsverband Deutscher Nervenärzte. http://www.bvdn.de/bvdn-themen/spitzenverband-bvdn.html [Abruf am: 11. Juni 2015].

Deutsche Gesellschaft für Palliativmedizin (2014a) Entwicklung der stationären Hospize und Palliativstationen einschl. der Einrichtungen für Kinder. Stand 10/2014. https://www.dgpalliativmedizin.de/images/stories/ Entwicklung_Palliativ_und_Hospiz_station\%C3\%A4r_1996-2014.JPG [Abruf am: 27. April 2015].

Deutsche Gesellschaft für Palliativmedizin (2014b) Spezialisierte ambulante Palliativversorgung (SAPV). http://www. dgpalliativmedizin.de/allgemein/sapv.html [Abruf am: 11. Juni 2015].
Deutscher Hospiz- und PalliativVerband e.V. (2015) Statistiken. http://www.dhpv.de/service_zahlen-fakten.html [Abruf am: 11. Juni 2015].

Deutscher Verband für Physiotherapie (ZVK) e.V. (2015a) Die Arbeitsgemeinschaften des Deutschen Verbandes für Physiotherapie (ZVK). Köln. https://www.physio-deutschland.de/fachkreise/verbandsstruktur/arbeitsgemeinschaften.html [Abruf am: 14. Dezember 2015].

Deutscher Verband für Physiotherapie (ZVK) e.V. (2015b) Unser Leitbild. Köln. https://www.physio-deutschland. de/der-bundesverband/philosophie.html [Abruf am: 14. Dezember 2015].

Deutscher Verband für Physiotherapie (ZVK) e.V. (2015c) Zahlen, Daten, Fakten aus berufsrelevanten Statistiken. Köln. https://www.physio-deutschland.de/fileadmin/ data/bund/Dateien_oeffentlich/Beruf_und_Bildung/ Zahlen_Daten_Falten/Zahlen_Daten_Fakten.pdf [Abruf am: 14. Dezember 2015].

DGBS e.V., DGPPN e.V. (2012) S3-Leitlinie zur Diagnostik und Therapie Bipolarer Störungen. Langversion 1.0. Deutsche Gesellschaft für Bipolare Störungen.

DGN (Hrsg.) (2014) Leitlinien für Diagnostik und Therapie in der Neurologie. Diagnose und Therapie der Multiplen Sklerose. Entwicklungsstufe: S2e. Stand: Januar 2012, Ergänzung August 2014. Gültig bis 2017. (AWMF-Registernummer: 030/050). Deutsche Gesellschaft für Neurologie. http://www.awmf.org/uploads/tx_szleitlinien/ 030-050l_S2e_Multiple_Sklerose_Diagnostik_Therapie_ 2014-08_verlaengert.pdf [Abruf am: 04. November 2015].

DGN - Deutsche Gesellschaft für Neurologie (2015) http:// www.dgn.org/ [Abruf am: 11. Juni 2015].

DGN, KKNMS (2014). Leitlinie zur Diagnose und Therapie der MS. Online-Version, Stand: 23.04.2014. Deutsche Gesellschaft für Neurologie, Kompetenznetz Multiple Sklerose. http://www.kompetenznetz-multiplesklerose.de/ images/stories/PDF_Dateien/Leitlinie/dgn-kknms_msII_20140423.pdf [Abruf am: 11. Juni 2015].

DGNR (2015) Geschäftsordnung für den Vorstand der Deutschen Gesellschaft für Neuroradiologie (DGNR). www.dgnr.org/media/document/173/Geschaeftsordnung-DGNR.pdf [Abruf am: 27. April 2015].

DGPPN (2015) Deutsche Gesellschaft für Psychiatrie und Psychotherapie, Psychosomatik und Nervenheilkunde. http:// www.dgppn.de/dgppn.html [Abruf am: 28. April 2015].

DGPPN, BÄK, KBV, AWMF, AkdÄ, BPtK, BApK, DAGSHG, DEGAM, DGPM, DGPS, DGRW (2015) S3-Leitlinie/Nationale VersorgungsLeitlinie Unipolare Depression. Langfassung, 2. Auflage 2015. AWMF-Register-Nr. nvl-005. Deutsche Gesellschaft für Psychiatrie und Psychotherapie, Psychosomatik und Nervenheilkunde. http://www. awmf.org/uploads/tx_szleitlinien/nvl-005I_Unipolare_ Depression_2015-12.pdf [Abruf am: 09. Februar 2016].

DMSG (2009) Unser neuer Weg: DMSG-geprüfte Fortbildung für Physiotherapeutinnen und -therapeuten. Deutsche Multiple Sklerose Gesellschaft Bundesverband e.V. http:// www.dmsg.de/dokumentearchiv/physiotherapie.pdf [Abruf am: 14. Dezember 2015]. 
DMSG (2012) Stichwort Patientenbeteiligung: DMSG-Bundesverband vertritt die Interessen von Multiple Sklerose Erkrankten im G-BA. Deutsche Multiple Sklerose Gesellschaft Bundesverband e.V. http://www.dmsg.de/ multiple-sklerose-news/index.php?w3pid=news\&kategorie $=$ rechtsfragen \&anr $=4548 \&$ suchbegriffe $=Z u-$ satznutzen\%20G-BA [Abruf am: 11. Juni 2015].

DMSG (2014) DMSG startet Beratungs-Hotline für schwer an Multipler Sklerose erkrankte Menschen. Deutsche Multiple Sklerose Gesellschaft Bundesverband e.V. http:// www.dmsg.de/multiple-sklerose-news/index. php? w3pid $=$ news\&kategorie $=$ aktuellesms \&anr $=5110$ [Abruf am: 11. Juni 2015].

DMSG (2015a) Aufgaben und Zielsetzung. Deutsche Multiple Sklerose Gesellschaft Bundesverband e.V. https://www. dmsg.de/dmsg-bundesverband/index.php?w3pid=dmsg [Abruf am: 11. Juni 2015].

DMSG (2015b) Bundesmodell »Pflege bei Multipler Sklerose» (MS). Deutsche Multiple Sklerose Gesellschaft Bundesverband e.V. http://www.dmsg.de/multiple-sklerose-news/ index.php?w3pid=news\&kategorie=aktuelles\&kategorie2=uebersicht [Abruf am: 11. Juni 2015].

DMSG (2015c) Daten und Fakten. Deutsche Multiple Sklerose Gesellschaft Bundesverband e.V. https://www.dmsg.de/ dmsg-bundesverband/index.php?w3pid=dmsg\&kategorie $=$ wirueberuns\&kategorie $2=$ datenundfakten $[$ Abruf am: 11. Juni 2015].

DMSG (2015d) Lebensbegleitende Beratung von MS-Betroffenen. Deutsche Multiple Sklerose Gesellschaft Bundesverband e.V. http://www.dmsg-berlin.de/beratung/ peer-counseling.html [Abruf am: 11. Juni 2015].

DMSG (2015e) Verzeichnis der Kliniken/Praxen. Deutsche Multiple Sklerose Gesellschaft Bundesverband e.V. http:// www.dmsg.de/service/index.php?w3pid=service\&kategorie $=$ mskliniken $\&$ kategorie $2=$ klinikenverzeichnis $[$ Abruf am: 22. Januar 2016].

DMSG (2016a) Neues im Gesundheitswesen 2016 - auch für MS-Erkrankte. Deutsche Multiple Sklerose Gesellschaft Bundesverband e.V. http://www.dmsg.de/ multiple-sklerose-news/index.php?w3pid=news\&kategorie $=$ rechtsfragen\&anr=5819 [Abruf am: 19. Januar 2016].

DMSG (2016b) Zentren. Deutsche Multiple Sklerose Gesellschaft Bundesverband e.V. http://www.dmsg.de/ msregister/index.php?nav=zentren [Abruf am: 22. Januar 2016].

DMSG Berlin e.V. (2015) Rehabilitation. Berlin. http://www. dmsg-berlin.de/multiple-sklerose/rehabilitation.html [Abruf am: 14. Dezember 2015].

DMSKW (2015) Deutschsprachiges Multiple Sklerose und Kinderwunsch Register. http://www.ms-und-kinderwunsch.de/ (Abruf am 06. August 2015).

Europäische Arzneimittel-Agentur (2015) European public assessment reports. http://www.ema.europa.eu/ema/ (Abruf am 06. August 2015).

Faller H (2012) Patientenorientierte Kommunikation in der Arzt-Patient-Beziehung. Bundesgesundheitsblatt Ge- sundheitsforschung Gesundheitsschutz 55(9), 1106-1112. DOI: 10.1007/s00103-012-1528-x.

Flachenecker P, Stuke K, Elias W, Freidel M, Haas J, PitschnauMichel D, Schimrigk S, Zettl UK, Rieckmann P (2008) Multiple sclerosis registry in Germany: results of the extension phase 2005/2006. Deutsches Ärzteblatt 105(7), 113-119. DOI: 10.3238/arztebl.2008.0113.

FobiZe (2015) Multiple Sklerose. Köln. http://www.fobize.de/ kurs_detail.php5?kennung=10610030 [Abruf am: 14 . Dezember 2015].

Galushko M, Golla H, Strupp J, Karbach U, Kaiser C, Ernstmann N, Pfaff H, Ostgathe C, Voltz R (2014) Unmet needs of patients feeling severely affected by multiple sclerosis in Germany: a qualitative study. J Palliat Med 17(3), 274-281. DOI: $10.1089 /$ jpm.2013.0497.

Gemeinsamer Bundesausschuss (2010) Der Gemeinsame Bundesausschuss stellt sich vor. https://www.g-ba.de/ downloads/17-98-2803/2010-01-01-Faltblatt-GBA.pdf [Abruf am: 27. April 2015].

Gemeinsamer Bundesausschuss (2014a) Nutzenbewertungsverfahren zum Wirkstoff Dimethylfumarat. https://www. g-ba.de/informationen/nutzenbewertung/111/ [Abruf am: 11. Juni 2015].

Gemeinsamer Bundesausschuss (2014b) Richtlinie des Gemeinsamen Bundesausschuss über die ambulante spezialfachärztliche Versorgung nach § $116 \mathrm{~b}$ SGB V (Richtlinie ambulante spezialfachärztliche Versorgung § 116b SGB V - ASV-RL). Stand: 20. Februar 2014. https:// www.g-ba.de/downloads/62-492-907/ASV-RL_2014-0220.pdf [Abruf am: 27. April 2015].

Gemeinsamer Bundesausschuss (2015a). https://www.g-ba. de/ [Abruf am: 11. Juni 2015].

Gemeinsamer Bundesausschuss (2015b) Ambulante spezialfachärztliche Versorgung nach $\S 116 \mathrm{~b}$ SGB V. https:// www.gba.de/institution/themenschwerpunkte/116b/ (Abruf am 04. August 2015).

Gemeinsamer Bundesausschuss (2015c) Aufgabe. https:// www.g-ba.de/institution/aufgabe/aufgabe/ [Abruf am: 11. Juni 2015].

Gemeinsamer Bundesausschuss (2015d) Der Gemeinsame Bundesausschuss stellt sich vor. https://www.g-ba.de/ institution/struktur/ [Abruf am: 11. Juni 2015].

Gemeinsamer Bundesausschuss (2015e) Tragende Gründe zum Beschluss des Gemeinsamen Bundesausschusses über eine Änderung der Arzneimittel-Richtlinie (AM-RL) Anlage XII - Beschlüsse über die Nutzenbewertung von Arzneimitteln mit neuen Wirkstoffen nach § 35a GB V - Fingolimod. https://www.g-ba.de/downloads/40-2683380/2015-10-01_AM-RL-XII_2015-04-01-D-157_Fingolimod-Abl-Befr_TrG.pdf [Abruf am: 19. Oktober 2015].

Gemeinsamer Bundesausschuss (2015f) Übersicht der Wirkstoffe. https://www.g-ba.de/informationen/nutzenbewertung/ [Abruf am: 11. Juni 2015].

Gerst T (2015) Ambulante spezialfachärztliche Versorgung: Wenig Euphorie bei den Beteiligten. Deutsches Ärzteblatt 112(6), 255. http://www.aerzteblatt.de/archiv/ 170899/Ambulante-spezialfachaerztliche-Versorgung- 
Wenig-Euphorie-bei-den-Beteiligten [Abruf am: 20. Oktober 2015].

Gesellschaft für Neuropsychologie (GNP) e.V. (2015a) Fachinformationen. 22.09.2015. 36001 Fulda: Gesellschaft für Neuropsychologie (GNP) e.V. http://www.gnp.de/_de/ fs-Info-fuer-Patienten.php [Abruf am: 27. Oktober 2015].

Gesellschaft für Neuropsychologie (GNP) e.V. (2015b) Postgraduale Weiterbildung Klinischer Neuropsychologe. 22.09.2015. 36001 Fulda: Gesellschaft für Neuropsychologie (GNP) e.V. http://www.gnp.de/_de/aw-WeiterbildungKNP.php [Abruf am: 27. Oktober 2015].

GKV Spitzenverband (Hrsg.) (2015) Faktenblatt: Thema: Ambulante Versorgung - Systematik Ärztehonorare. https:// www.gkv-spitzenverband.de/media/dokumente/presse/ presse_themen/aerzteverguetung/Faktenblatt_Systematik_Aerztehonorare_2015-04-28.pdf [Abruf am: 20. Oktober 2015].

heimerer akademie (2015) MS - Neurorehabilitation bei Multipler Sklerose. Landsberg am Lech. https://www.heimererakademie.de/nc/physiotherapieosteopathie/kursbe schreibung/?tid=13438\&sid=3\&kbid=1091\&nid=6\& $c d f d=1453503600$ [Abruf am: 14. Dezember 2015].

IGES Institut (2014) Neurologische und psychiatrische Versorgung aus sektorenübergreifender Perspektive. Studie im Auftrag von Berufsverband Deutscher Nervenärzte e.V. (BVDN), Berufsverband Deutscher Neurologen e.V. (BDN), Berufsverband Deutscher Psychiater e.V (BVDP), Kassenärztliche Bundesvereinigung (KBV) / Zentralinstitut für die kassenärztliche Versorgung in Deutschland (ZI), Deutsche Gesellschaft für Neurologie e.V. (DGN). Ergebnisbericht. Berlin: IGES Institut.

KBV (2012) Thema: Neuropsychologische Therapie: Information der KBV 39/2012. Kassenärztliche Bundesvereinigung. https://www.kvno.de/downloads/quali/praxisinfo_neuropsychologische_therapie.pdf [Abruf am: 20. Oktober 2015].

KVB (2013) Praxisbesonderheiten und langfristiger Heilmittelbedarf: Hinweise und Erläuterungen zu der neuen Vereinbarung in der Heilmittelversorgung. München. https://www.kvb.de/fileadmin/kvb/dokumente/Praxis/ Infomaterial/Verordnung/KVB-Broschuere-Praxisbesonderheiten-langfristiger-Heilmittelbedarf.pdf [Abruf am: 14. Dezember 2015].

Kienle G (2008) Evidenzbasierte Medizin und ärztliche Therapiefreiheit: Vom Durchschnitt zum Individuum. Deutsches Ärzteblatt 25(105). http://www.aerzteblatt.de/ archiv/60581/Evidenzbasierte\%ADMedizin\%ADund\% ADaerztliche\%ADTherapiefreiheit\%ADVom\%ADDurchschnitt\%ADzum\%ADIndividuum4/4 [Abruf am: 19. Oktober 2015].

Kliniken der Stadt Köln gGmbH (2015) Klinik für Neurologie Köln-Merheim engagiert sich im Projekt »Integrierte Versorgung Multiple Sklerose (IGV-MS Rheinland)«. http://www.kliniken-koeln.de/Merheim_Neurologie Schwerpunkt_MS_Integrierte_Versorgung.htm [Abruf am: 11. Juni 2015].
Kompetenznetz Multiple Sklerose (2015a) Besser informieren - Zielsicher entscheiden - Erfolgreich behandeln. http://www.kompetenznetz-multiplesklerose.de/de/ kohorte- studien-infrastruktur/decims [Abruf am: 11. Juni 2015].

Kompetenznetz Multiple Sklerose (2015b) Gemeinsame Richtlinien für den Umgang mit Biomaterial. http://www. kompetenznetz-multiplesklerose.de/de/kohorte-studieninfrastruktur/biobanking [Abruf am: 11. Juni 2015].

Kompetenznetz Multiple Sklerose (2015c) Immuntherapieregister für mehr Arzneimittelsicherheit in der MS-Therapie. http://www.kompetenznetz-multiplesklerose.de/de/ kohorte-studien-infrastruktur/regims [Abruf am: 11. Juni 2015].

Kompetenznetz Multiple Sklerose (2015d) Patientenstudie soll neue Krankheitsmerkmale identifizieren. http://www. kompetenznetz-multiplesklerose.de/de/kohorte-studieninfrastruktur/kohortenstudie [Abruf am: 11. Juni 2015].

Kompetenznetz Multiple Sklerose (2015e) Ursache und Entstehung ergründen - Neue Therapien entwickeln. http:// www.kompetenznetz-multiplesklerose.de/forschung [Abruf am: 11. Juni 2015].

Landesportal Schleswig-Holstein (2015) Krankenhäuser Liste der Zulassungen. http://www.schleswig-holstein. de/DE/Fachinhalte/K/krankenhaeuser/Downloads/ krankenhaeuser_listeZulassungen.pdf?_blob=publicationFile\&v=1 [Abruf am: 11. Juni 2015].

Landeszentrum Gesundheit Nordrhein-Westfalen (2015) Integrierte Versorgung von Patienten mit MS im Rheinland Deutsche Multiple Sklerose Gesellschaft, Landesverband NRW e.V. http://www.kliniken-koeln.de/upload/ infoportal_241256_Landeszentrum_Gesundheit_NRW_ Multiple_Sklerose_8792.pdf [Abruf am: 30. April 2015].

Limmroth V, Meier U, Nelles G (2006) Integrierte Versorgung Multiple Sklerose. Ein »lernendes « und »plastisches « System. Info Neurologie und Psychiatrie 8 (Sonderheft Nr. 1).

MSK (2015) Initiative Selbsthilfe Multiple Sklerose Kranker e.V. http://www.multiple-sklerose-e-v.de/ueberuns/mskev. html [Abruf am: 11. Juni 2015].

Nelles G, Limmroth V, Faber B, May M, Schipper S, Meier U, Pöhlau D (2013) Multple Sklerose. Was bringt die integrierte Versorgung wirklich? NeuroTransmitter 24(5), 34-43.

Nelles G, Meier U, Limmroth V, Pöhlau D, Wirtz M, Münscher C, Faber B (2010) Integrierte Versorgung Multiple Sklerose - Modellregion Nordrhein. 2-Jahres-Verlaufsbeobachtung. Aktuelle Neurologie 37, 1-8. DOI: 10.1055/s-00301248480

Patti F (2010) Optimizing the benefit of multiple sclerosis therapy: the importance of treatment adherence. Patient preference and adherence 4, 1-9. ISSN: 1177-889X.

Pfaff $\mathrm{H}$ (2003) Versorgungsforschung - Begriffsbestimmung, Gegenstand und Aufgaben. In: Pfaff H, Schrappe M, Lauterbach KW, Engelmann U, Halber M: Gesundheitsversorgung und Disease Management. Grundlagen und Anwendungen der Versorgungsforschung. Bern: Verlag Hans Huber, 13-23. ISBN: 978-3456840260. 
Pfaff H, Neugebauer EAM, Glaeske G, Schrappe M (2011) Lehrbuch Versorgungsforschung: Systematik - Methodik - Anwendung. Stuttgart: Schattauer GmbH. ISBN: 978-37945-2797-7.

Remington G, Rodriguez Y, Logan D, Williamson C, Treadaway K (2013) Facilitating medication adherence in patients with multiple sclerosis. International journal of MS care 15(1), 36-45. DOI: 10.7224/1537-2073.2011-038.

Rupprecht C, Schulte C (2013) Patienten und Versicherten eine Stimme geben. Forschungsjournal Soziale Bewegungen 26(2), 149-151. http://forschungsjournal.de/ jahrgaenge/2013heft2 [Abruf am: 1. Februar 2016].

Sachverständigenrat zur Begutachtung der Entwicklung im Gesundheitswesen (2014) Bedarfsgerechte Versorgung - Perspektiven für ländliche Regionen und ausgewählte Leistungsbereiche. http://www.svr-gesundheit.de/file admin/user_upload/Gutachten/2014/SVR-Gutachten_ 2014_Langfassung.pdf [Abruf am: 30. April 2015].

Schlenker R-U (2014) Defizite in der MS-Versorgung? Was können die Kassen tun? [Vortrag] 9. Medizinkongress der BARMER GEK und der Universität Bremen 24. Juni 2014. Berlin. http://www.zes.uni-bremen.de/uploads/Veranstaltungen/2014/140624_Medizinkongress_Vortrag_ Schlenker.pdf [Abruf am: 30. April 2015].

Schlingensiepen I (2010) Integrierte Versorgung hilft MS-Patienten. Ärzte Zeitung 04.11.2010. http://www.aerztezeitung.de/politik_gesellschaft/krankenkassen/article/ 626891/integrierte-versorgung-hilft-ms-patienten.html [Abruf am: 11. Juni 2015].

Schlingensiepen I (2014) MS-Patienten in Kompetenzzentren behandeln. Ärzte Zeitung 15.01.2014. http://www.aerztezeitung.de/politik_gesellschaft/versorgungsforschung/?s $\mathrm{id}=852847$ [Abruf am: 11. Juni 2015].

Senatsverwaltung für Gesundheit und Soziales Berlin (2013) Ambulanten Behandlung im Krankenhaus nach § $116 \mathrm{~b}$ Abs. 2 SGB V - Zugelassene Krankenhäuser in Berlin (mit bestandskräftigen Bescheiden). Stand: 24.04.2013. http:// www.berlin.de/sen/gesundheit/_assets/themen/ stationaere-versorgung/zugelassene_kh_nach 116b. pdf. [Abruf am: 30. April 2015].

Staatsministerium für Soziales und Verbraucherschutz Freistaat Sachsen (2011) Zugelassene Krankenhäuser nach $\S 116 b$ SGB V, Stand: November 2011. http://www.gesunde.sachsen.de/download/Download_Gesundheit/ Tabelle_Zugelassene_Krankenhaeuser_in_Sachsen.pdf [Abruf am: 30. April 2015].

Universitätsmedizin Göttingen, Institut für Multiple-Sklerose-Forschung, Geschichte des Instituts für Multiple-Sklerose-Forschung (IMSF). http://www.neuroimmunologie. uni-goettingen.de/?id=8 [Abruf am: 11. Juni 2015].

Verband Forschender Arzneimittelhersteller e.V. (2015) Forschungsschwerpunkte der vfa-Mitgliedsunternehmen. http://www.vfa.de/de/arzneimittel-forschung/ forschungsschwerpunkte?c=Multiple+Sklerose [Abruf am: 11. Juni 2015].
Weegen L, Korff L, Mostardt S, Ivancevic S, Wasem J, Walendzik A (2013) Bestandsaufnahme zu Versorgungsdefiziten und Versorgungsmanagementprogrammen bei Multiple Sklerose in Deutschland. Das Gesundheitswesen 75 A193. DOI: 10.1055/s-0033-1354151.

Windt R (2014) Multiple Sklerose - Neue Therapieoptionen. In: Glaeske G, Schicktanz C: BARMER GEK Arzneimittelreport 2014. Auswertungsergebnisse der BARMER GEK Arzneimitteldaten aus den Jahren 2012 bis 2013. BARMER GEK.

Wirtz M (2010) Integrierte Versorgung (IV) der Multiplen Sklerose in Nordrhein-Westfalen. In: Amelung V, Bergmann F, Falkei P, Hauth I, Jaleel E, Meier U, Reichmann H, Roth-Sackenheim C: Innovative Konzepte im Versorgungsmanagement von ZNS-Patienten. Berlin: MWV Medizinisch Wissenschaftliche Verlagsgesellschaft, 155. ISBN: 9783941468184.

Ziegler K (2007) Evidenzbasierte Physiotherapie bei Multipler Sklerose. Nervenheilkunde 26(12), 1088-1094. ISSN: 07221541.

Ziemssen T, Kern R, Cornelissen C (2015) The PANGAEA study design - a prospective, multicenter, non-interventional, long-term study on fingolimod for the treatment of multiple sclerosis in daily practice. BMC Neurology 15:39. DOI: 10.1186/s12883-015-0342-0. http://www.biomedcentral.com/1471-2377/15/93 [Abruf am: 15. Dezember 2015]. 